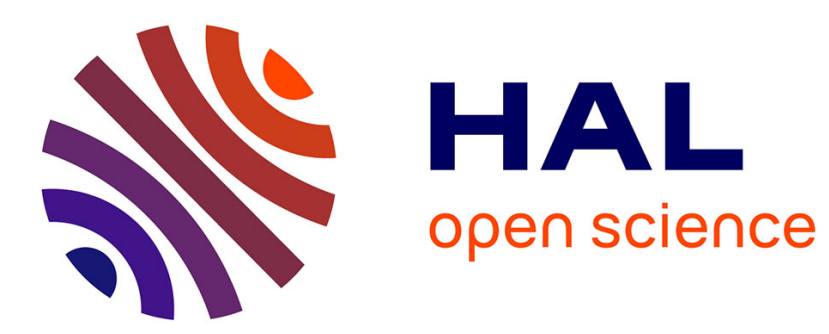

\title{
Finite volume implementation of the method of asymptotic partial domain decomposition for the heat equation on a thin structure
}

Grigory Panasenko, Marie-Claude Viallon

\section{- To cite this version:}

Grigory Panasenko, Marie-Claude Viallon. Finite volume implementation of the method of asymptotic partial domain decomposition for the heat equation on a thin structure. Russian Journal of Mathematical Physics, 2015, 22 (2), pp.237-263. 10.1134/S1061920815020107 . hal-01223472

HAL Id: hal-01223472

https://hal.science/hal-01223472

Submitted on 2 Nov 2015

HAL is a multi-disciplinary open access archive for the deposit and dissemination of scientific research documents, whether they are published or not. The documents may come from teaching and research institutions in France or abroad, or from public or private research centers.
L'archive ouverte pluridisciplinaire HAL, est destinée au dépôt et à la diffusion de documents scientifiques de niveau recherche, publiés ou non, émanant des établissements d'enseignement et de recherche français ou étrangers, des laboratoires publics ou privés. 


\title{
FINITE VOLUME IMPLEMENTATION OF THE METHOD OF ASYMPTOTIC PARTIAL DOMAIN DECOMPOSITION FOR THE HEAT EQUATION ON A THIN STRUCTURE *
}

\author{
GRIGORY PANASENKO ${ }^{\dagger}$ AND MARIE-CLAUDE VIALLON
}

\begin{abstract}
The non-steady heat equation is considered in thin structures. The asymptotic expansion of the solution constructed earlier is used for evaluation of the partial derivatives of the solution. The method of partial asymptotic domain decomposition is applied to the non-steady heat equation. It reduces the original $2 \mathrm{D}$ model to a hybrid dimension one, partially $2 \mathrm{D}$, partially $1 \mathrm{D}$ with some special interface conditions between $2 \mathrm{D}$ and $1 \mathrm{D}$ parts. The finite volume method is applied for the numerical solution of the hybrid dimension model. The error estimate is proved. The numerical experiment confirms the theoretical error evaluation.
\end{abstract}

Key words. heat equation, thin structures, asymptotics, partial domain decomposition, finite volume scheme, parabolic problem

AMS subject classifications. 35B27, 35Q53, 35C20, 35J25, 65N12, 76M12

1. Introduction. The method of asymptotic partial decomposition for thin structures was proposed in [5], and then developed in [6]. Thin rod structures are connected finite unions of thin finite cylinders (in the $2 D$ case respectively thin rectangles) where the ratio of the diameter and the hight of the cylinders is the small parameter $\varepsilon$. Each such structure may be schematically represented by its graph: letting the thickness of cylinders to zero we find out that cylinders degenerate to segments. Although the method is developed for the steady problems, there are only few examples of its application to the non-steady equations (see [8]). In the present paper the heat equation set on the thin structure with the Neumann boundary condition at the lateral boundary is considered. An asymptotic expansion of the solution to the problem was constructed in [7]. It has a regular part, expansion in powers of $\varepsilon$ with coefficients depending on the time variable and the longitudinal space variable only, and the boundary layer correctors depending on the dilated space variables $x / \varepsilon$ and on the time and decaying exponentially with respect to the space variables, so that their values at some small distance from the bases of the cylinders become of order of $\varepsilon^{J}$ for any $J$. This property of the asymptotic expansion allows us to "cut " the cylinders at the distance of order $\varepsilon|\ln (\varepsilon)|$ from the bases of the cylinders, to reduce dimension in the truncated middle parts of the cylinders and to set at the truncated sections some special asymptotically justified interface conditions between the $1 \mathrm{D}$ and multi-dimensional parts (see [5]). Note that in the non-steady case these conditions are the same as in the steady case [5].

A finite volume scheme is applied for the numerical solution of this hybrid dimension problem. A specific discrete $H^{1}$ norm is defined that allows to prove an error estimate for the finite volume scheme. The numerical experiments confirm the theoretical error evaluation. The finite volume scheme for the steady state hybrid dimension problem was studied in [9], [10].

2. Graphs. Let $O_{1}, O_{2}, \ldots, O_{N}$ be $N$ different points in $\mathbb{R}^{n}, n=2,3$, and $e_{1}, e_{2}, \ldots, e_{M}$ be $M$ closed segments each connecting two of these points (i.e. each

\footnotetext{
*Université de Lyon, Institut Camille Jordan, UMR CNRS 5208, Faculté des Sciences et Techniques, 23 rue Docteur Paul Michelon, 42023 Saint-Etienne Cedex 2, France.

$\dagger$ (Grigory.panasenko@univ-st-etienne.fr).
} 
$e_{j}=\left[O_{i_{j}}, O_{k_{j}}\right]$, where $\left.i_{j}, k_{j} \in\{1, \ldots, N\}, i_{j} \neq k_{j}\right)$. All points $O_{i}$ are supposed to be the ends of some segments $e_{j}$. The segments $e_{j}$ are called edges of the graph. A point $O_{i}$ is called node if it is the common end of at least two edges and $O_{i}$ is called vertex if it is the end of the only one edge. Any two edges $e_{j}$ and $e_{i}$ can intersect only at the common node. The set of vertices is supposed to be non-empty.

Denote $\mathcal{B}=\bigcup_{j=1}^{M} e_{j}$ the union of edges and assume that $\mathcal{B}$ is a connected set. The graph $\mathcal{G}$ is defined as the collection of nodes, vertices and edges.

The union of all edges having the same end point in $O_{l}$ is called the bundle $\mathcal{B}^{(l)}$.

Let $e$ be some edge, $e=\left[O_{i}, O_{j}\right]$. Consider two Cartesian coordinate systems in $\mathbb{R}^{n}$. The first one has the origin in $O_{i}$ and the axis $O_{i} x_{1}^{(e)}$ has the direction of the ray $\left[O_{i} O_{j}\right)$; the second one has the origin in $O_{j}$ and the opposite direction, i.e. $O_{i} \tilde{x}_{1}^{(e)}$ is directed over the ray $\left[O_{j} O_{i}\right)$.

Further in various situations we will chose one or another coordinate system denoting the local variable in both cases as $\left.x^{(} e\right)$ and pointing out which end is taken as the origin of the coordinate system.

3. Rod structures. With every edge $e_{j}$ we associate a bounded domain $\sigma_{j} \subset$ $\mathbb{R}^{n-1}$ having $C^{4}$-smooth boundary $\partial \sigma_{j}, j=1, \ldots, M$. For every edge $e_{j}=e$ and associated $\sigma_{j}=\sigma^{(e)}$ we denote by $B_{\varepsilon}^{(e)}$ the cylinder

$$
B_{\varepsilon}^{(e)}=\left\{x^{(e)} \in \mathbb{R}^{n}: x_{1}^{(e)} \in(0,|e|), \frac{x^{(e) \prime}}{\varepsilon} \in \sigma^{(e)}\right\},
$$

where $x^{(e) \prime}=\left(x_{2}^{(e)}, \ldots, x_{n}^{(e)}\right),|e|$ is the length of the edge $e$ and $\varepsilon>0$ is a small parameter. Notice that the edges $e_{j}$ and Cartesian coordinates of nodes and vertices $O_{j}$, as well as domains $\sigma_{j}$, do not depend on $\varepsilon$.

Let $O_{1}, \ldots, O_{N_{1}}$ be nodes and $O_{N_{1}+1}, \ldots, O_{N}$ be vertices. Let $\omega^{1}, \ldots, \omega^{N_{1}}$ be bounded independent of $\varepsilon$ domains in $\mathbb{R}^{n}$ with Lipschitz boundaries $\partial \omega^{j}$; introduce the nodal domains $\omega_{\varepsilon}^{j}=\left\{x \in \mathbb{R}^{n}: \frac{x-O_{j}}{\varepsilon} \in \omega^{j}\right\}$.

Every vertex $O_{j}$ is the end of one and only one edge $e_{k}$. By a rod structure we call the following domain

$$
B_{\varepsilon}=\left(\bigcup_{j=1}^{M} B_{\varepsilon}^{\left(e_{j}\right)}\right) \cup\left(\bigcup_{j=1}^{N_{1}} \omega_{\varepsilon}^{j}\right)
$$

Assume that it is a connected set and that the boundary $\partial B_{\varepsilon}$ of $B_{\varepsilon}$ is $C^{4}$-smooth except for the boundary of the bases of cylinders $\gamma_{\varepsilon}^{i}$ containing the vertices $O_{i}, i=$ $N_{1}+1, \ldots, N$. Without loss of generality assume that

$$
\left(B_{\varepsilon}^{\left(e_{j}\right)} \backslash\left(\bigcup_{i=1}^{N_{1}} \omega_{\varepsilon}^{i}\right)\right) \cap\left(B_{\varepsilon}^{\left(e_{k}\right)} \backslash\left(\bigcup_{i=1}^{N_{1}} \omega_{\varepsilon}^{i}\right)\right)=\emptyset
$$

for $j \neq k$. Denote $\gamma_{\varepsilon}=\bigcup_{i=N_{1}+1}^{N} \gamma_{\varepsilon}^{i}$. 
4. Formulation of the heat equation in rod structure. Consider the initial boundary value problem for the non-steady heat equations in the tube structure $B_{\varepsilon}$

$$
\begin{gathered}
\frac{\partial u_{\varepsilon}}{\partial t}-\Delta u_{\varepsilon}=f(x, t), \quad x \in B_{\varepsilon}, t \in(0, T), \\
\frac{\partial u_{\varepsilon}}{\partial n}=0, \quad x \in \partial B_{\varepsilon} \backslash \gamma_{\varepsilon}, t \in(0, T), \\
u_{\varepsilon}=0, \quad x \in \gamma_{\varepsilon}, t \in(0, T) \\
u_{\varepsilon}(x, 0)=0, x \in B_{\varepsilon} .
\end{gathered}
$$

The right hand side $f$ is a function defined on $B_{\varepsilon} \times[0, T]$ such that $f(x, t)=$ $f_{j}\left(x_{1}^{(e)}, t\right)$, if $x \in B_{\varepsilon}^{\left(e_{j}\right)}, \quad j=1, \ldots, M$, where $f_{j}$ are independent of $\varepsilon C^{J+4}$-smooth functions and they are constant with respect to $x$ in some neighborhood of the nodes and vertices. The values of $f$ in the domains $\omega_{\varepsilon}^{i}$ are equal to its value in the node or vertex $O_{i}$. We assume that $f_{j}(., t)=0$ for $t \leq t_{0}, t_{0}>0$.

The variational formulation of problem (4.1) is : find $u_{\varepsilon} \in H_{\gamma, 0}^{1}\left(B_{\varepsilon} \times(0, T)\right)$ such that for almost all $t \in(0, T)$,

$$
\begin{gathered}
\int_{B_{\varepsilon}}\left(\frac{\partial u_{\varepsilon}}{\partial t} v+\nabla u_{\varepsilon} \cdot \nabla v\right) d x=\int_{B_{\varepsilon}} f v d x, \quad v \in H_{\gamma, 0}^{1}\left(B_{\varepsilon}\right), \\
\left.u_{\varepsilon}\right|_{t=0}=0,
\end{gathered}
$$

where

$$
\begin{aligned}
H_{\gamma, 0}^{1}\left(B_{\varepsilon}\right) & =\left\{v \in H^{1}\left(B_{\varepsilon}\right)|v|_{\gamma_{\varepsilon}}=0\right\}, \\
H_{\gamma, 0}^{1}\left(B_{\varepsilon} \times(0, T)\right) & =\left\{v \in H^{1}\left(B_{\varepsilon} \times(0, T)\right)|v|_{\gamma_{\varepsilon}}=0\right\} .
\end{aligned}
$$

This variational formulation implies:

(4.4) $\int_{B_{\varepsilon} \times(0, T)}\left(\frac{\partial u_{\varepsilon}}{\partial t} v+\nabla u_{\varepsilon} \cdot \nabla v\right) d x d t=\int_{B_{\varepsilon} \times(0, T)} f v d x d t, v \in H_{\gamma, 0}^{1,0}\left(B_{\varepsilon} \times(0, T)\right)$,

$$
\left.u_{\varepsilon}\right|_{t=0}=0
$$

where

$$
\begin{gathered}
H_{\gamma, 0}^{1,0}\left(B_{\varepsilon} \times(0, T)\right) \\
=\left\{v \in L^{2}\left(B_{\varepsilon} \times(0, T)\right)\left|\|u\|_{L^{2}\left(B_{\varepsilon} \times(0, T)\right)}+\|\nabla u\|_{L^{2}\left(B_{\varepsilon} \times(0, T)\right)}<+\infty, v\right|_{\gamma_{\varepsilon}}=0\right\} .
\end{gathered}
$$
holds

$$
\left\|u_{\varepsilon}\right\|_{H^{1}\left(B_{\varepsilon} \times(0, T)\right)} \leq C_{P F}\|f\|_{L^{2}\left(B_{\varepsilon} \times(0, T)\right)}
$$

where the constant $C_{P F}$ is independent of $\varepsilon$.

Proof: The proof of the theorem is based on the Galerkin method (see [7]) and the Poincaré-Friedrichs inequality for the rod structures with the constant independent of $\varepsilon$ ([6] Chapter 4, Appendices).

REMARK 1. This estimate (4.6) holds in the case if the right hand side is any function of $L^{2}\left(B_{\varepsilon} \times(0, T)\right)$ free of the above regularity restrictions. 
5. Construction of an asymptotic expansion of the solution. Let us seek the $J$-th approximation of an asymptotic expansion of the solution to problem (4.1) in the form:

$$
\begin{aligned}
u_{\varepsilon}^{(J)}= & \sum_{j=1}^{M} \zeta\left(\frac{x_{1}^{\left(e_{j}\right)}}{3 r \varepsilon}\right) \zeta\left(\frac{\left|e_{j}\right|-x_{1}^{\left(e_{j}\right)}}{3 r \varepsilon}\right) v_{j}\left(x_{1}^{\left(e_{j}\right)}, t\right) \chi_{j}\left(x_{1}^{\left(e_{j}\right)}\right)+ \\
& +\sum_{i=1}^{N_{1}} V_{i}^{B L}\left(\frac{x-O_{i}}{\varepsilon}, t\right)\left(1-\zeta\left(\frac{x-O_{i}}{e_{\min }}\right)\right)
\end{aligned}
$$

where $r$ is the maximal diameter of domains $\omega_{j}, \zeta$ is a smooth cut-off function independent of $\varepsilon$ with $\zeta(\tau)=0$ for $\tau \leq 1 / 3, \zeta(\tau)=1$ for $\tau \geq 2 / 3,0 \leq \zeta(\tau) \leq 1$; $e_{\text {min }}$ is the minimal length of the edges; $\chi_{j}\left(x_{1}^{\left(e_{j}\right)}\right)=1$ iff $x_{1}^{\left(e_{j}\right)} \in\left(0,\left|e_{j}\right|\right)$, and it is equal to zero if not; functions $v_{j}$ satisfy the heat equation on the graph $\mathcal{G}$ with some Kirchhoff-type junction conditions in the nodes $O_{i}, i=1, \ldots, N_{1}$, and the Dirichlet condition in the vertices $O_{i}, i=N_{1}, \ldots, N ; V_{i}^{B L}, i=1, \ldots, N$, are the boundary layer correctors. If $e_{j}$ is the edge with a vertex as one of the end points, then the local coordinate $\xi_{1}^{\left(e_{j}\right)}$ is directed from the node to the vertex and the factor $\zeta\left(\frac{\left|e_{j}\right|-x_{1}^{\left(e_{j}\right)}}{3 r \varepsilon}\right)$ is omitted.

Let us specify now $v_{j}$ and $V_{i}^{B L}$ :

$$
\begin{aligned}
v_{j}\left(x_{1}^{\left(e_{j}\right)}, t\right) & =\sum_{l=0}^{J} \varepsilon^{l} v_{j l}\left(x_{1}^{\left(e_{j}\right)}, t\right), \\
V_{i}^{B L}(\xi, t) & =\sum_{l=0}^{J} \varepsilon^{l} V_{i l}^{B L}(\xi, t) .
\end{aligned}
$$

Substituting the first term of the expansion into the equation, we get the residual which has to be compensated by the boundary layer correctors. This residual has the form:

$$
\begin{gathered}
\sum_{j=1}^{M}\left(\frac{\partial}{\partial t}-\left(\frac{\partial}{\partial x_{1}^{\left(e_{j}\right)}}\right)^{2}\right)\left\{\zeta\left(\frac{x_{1}^{\left(e_{j}\right)}}{3 r \varepsilon}\right) \zeta\left(\frac{\left|e_{j}\right|-x_{1}^{\left(e_{j}\right)}}{3 r \varepsilon}\right) v_{j}\left(x_{1}^{\left(e_{j}\right)}, t\right) \chi_{j}\left(x_{1}^{\left(e_{j}\right)}\right)\right\} \\
=\sum_{j=1}^{M}\left(\left(\frac{\partial v_{j}\left(\left(x_{1}^{\left(e_{j}\right)}, t\right)\right.}{\partial t}-\frac{\partial^{2} v_{j}\left(x_{1}^{\left(e_{j}\right)}, t\right)}{\left.\partial x_{1}^{\left(e_{j}\right)}\right)}\right) \zeta\left(\frac{x_{1}^{\left(e_{j}\right)}}{3 r \varepsilon}\right) \zeta\left(\frac{\left|e_{j}\right|-x_{1}^{\left(e_{j}\right)}}{3 r \varepsilon}\right)-\right. \\
-\frac{2}{\varepsilon} \frac{\partial v_{j}\left(x_{1}^{\left(e_{j}\right)}, t\right)}{\partial x_{1}^{\left(e_{j}\right)}} \frac{\partial}{\partial \xi_{1}^{\left(e_{j}\right)}}\left(\zeta\left(\frac{\xi_{1}^{\left(e_{j}\right)}}{3 r}\right) \zeta\left(\frac{\left|e_{j}\right|-\xi_{1}^{\left(e_{j}\right)}}{3 r}\right)\right)- \\
\left.\left.-\frac{1}{\varepsilon^{2}} v_{j}\left(x_{1}^{\left(e_{j}\right)}, t\right) \frac{\partial^{2}}{\partial \xi_{1}^{\left(e_{j}\right) 2}}\left(\zeta\left(\frac{\xi_{1}^{\left(e_{j}\right)}}{3 r}\right) \zeta\left(\frac{\left|e_{j}\right|-\xi_{1}^{\left(e_{j}\right)}}{3 r}\right)\right)\right)\left.\right|_{\xi^{\left(e_{j}\right)}=x^{\left(e_{j}\right)} / \varepsilon^{\left(x_{j}\right.}} \chi^{\left(e_{j}\right)}\right)=
\end{gathered}
$$

Remind that $v_{j}$ are defined in such a way that $\frac{\partial v_{j}}{\partial t}-\frac{\partial^{2} v_{j}}{\partial x_{1}^{\left(e_{j}\right) 2}}=f_{j}$, so that the first term of the sum is equal to 


$$
\left.\sum_{j=1}^{M} f_{j}\left(x_{1}^{\left(e_{j}\right)}, t\right)\right) \zeta\left(\frac{x_{1}^{\left(e_{j}\right)}}{3 r \varepsilon}\right) \zeta\left(\frac{\left|e_{j}\right|-x_{1}^{\left(e_{j}\right)}}{3 r \varepsilon}\right) \chi_{j}\left(x_{1}^{\left(e_{j}\right)}\right) .
$$

Note that $\left.f_{j}\left(x_{1}^{\left(e_{j}\right)}, t\right)\right)$ is a time dependent constant in every connected part of $\operatorname{supp}\left\{\zeta\left(\frac{x_{1}^{\left(e_{j}\right)}}{3 r \varepsilon}\right) \zeta\left(\frac{\left|e_{j}\right|-x_{1}^{\left(e_{j}\right)}}{3 r \varepsilon}\right)-1\right\}$.

These components are some neighborhoods of the extremities of the edge $e_{j}$. In particular, in the $e_{\min } / 2$-neighborhood of nodes and vertices $O_{i}$, we have:

$f_{j}\left(x_{1}^{\left(e_{j}\right)}, t\right)\left\{\zeta\left(\frac{x_{1}^{\left(e_{j}\right)}}{3 r \varepsilon}\right) \zeta\left(\frac{\left|e_{j}\right|-x_{1}^{\left(e_{j}\right)}}{3 r \varepsilon}\right)-1\right\}=f_{j}(0, t)\left\{\zeta\left(\frac{x_{1}^{\left(e_{j}\right)}}{3 r \varepsilon}\right) \zeta\left(\frac{\left|e_{j}\right|-x_{1}^{\left(e_{j}\right)}}{3 r \varepsilon}\right)-1\right\}$

and

$$
\begin{gathered}
\sum_{j=1}^{M} f_{j}\left(x_{1}^{\left(e_{j}\right)}, t\right) \zeta\left(\frac{x_{1}^{\left(e_{j}\right)}}{3 r \varepsilon}\right) \zeta\left(\frac{\left|e_{j}\right|-x_{1}^{\left(e_{j}\right)}}{3 r \varepsilon}\right) \chi_{j}\left(x_{1}^{\left(e_{j}\right)}\right) \\
=f(x, t)+\sum_{i=1}^{N_{1}} f\left(O_{i}, t\right)\left\{\sum_{j: O_{i} \in e_{j}} \zeta\left(\frac{x_{1}^{\left(e_{j}\right)}}{3 r \varepsilon}\right) \chi_{j}\left(x_{1}^{\left(e_{j}\right)}\right)-1\right\} \chi\left(\frac{\left|x-O_{i}\right|}{e_{\min }}\right),
\end{gathered}
$$

where $\chi(t)=1$ for $|t|<\frac{1}{2}, \chi(t)=0$ for $|t| \geq \frac{1}{2}$.

Let us expand now the functions $v_{j l}$ and $\frac{\partial v_{j l}}{\partial x_{1}^{\left(e_{j}\right)}}$ according to Taylor's formula

$$
\begin{gathered}
\left.\left.v_{j l}\left(x_{1}^{\left(e_{j}\right)}, t\right)\right)=v_{j l}(0, t)\right)+ \\
+\sum_{m=1}^{J-l} \varepsilon^{m} \frac{1}{m !} \frac{\partial^{m} v_{j l}}{\partial x_{1}^{\left(e_{j}\right) m}}(0, t) \xi_{1}^{\left(e_{j}\right) m}+\varepsilon^{J-l+1} \frac{1}{(J-l+1) !} \frac{\partial^{J-l+1} v_{j l}}{\partial x_{1}^{\left(e_{j}\right)(J-l+1)}}(\theta, t) \xi_{1}^{\left(e_{j}\right)(J-l+1)}
\end{gathered}
$$

and

$$
\begin{gathered}
\frac{\partial v_{j l}}{\partial x_{1}^{\left(e_{j}\right)}}\left(x_{1}^{\left(e_{j}\right)}, t\right)=\frac{\partial v_{j l}}{\partial x_{1}^{\left(e_{j}\right)}}(0, t)+ \\
+\sum_{m=1}^{J-l} \varepsilon^{m} \frac{1}{m !} \frac{\partial^{m+1} v_{j l}}{\partial x_{1}^{\left(e_{j}\right)(m+1)}}(0, t) \xi_{1}^{\left(e_{j}\right) m}+\varepsilon^{J-l+1} \frac{1}{(J-l+2) !} \frac{\partial^{J-l+2} v_{j l}}{\partial x_{1}^{\left(e_{j}\right)(J-l+2)}}(\theta, t) \xi_{1}^{\left(e_{j}\right)(J-l+1)}, \\
\xi^{\left(e_{j}\right)}=x^{\left(e_{j}\right)} / \varepsilon
\end{gathered}
$$

Then the residual (more exactly the result of the substitution) of the first sum of (5.1) in the $e_{\min } / 2$-neighborhood of nodes and vertices $O_{i}$ is finally equal to

$$
f(x, t)+\sum_{l=0}^{J} \varepsilon^{l-2} F_{i l}(\xi, t)+R_{J \varepsilon}(x, t)
$$


where $\xi=\left(x-O_{i}\right) / \varepsilon$,

$$
\begin{gathered}
F_{i l}(\xi, t)=-\left\{f\left(O_{i}, t\right)\left\{\sum_{j=1}^{M} \zeta\left(\frac{\xi_{1}^{\left(e_{j}\right)}}{3 r}\right) \psi\left(\xi_{1}^{\left(e_{j}\right)}\right)-1\right\} \delta_{l 2}\right. \\
+2 \sum_{m+p=l-1} \sum_{j=1}^{M}\left(\frac{1}{m !} \frac{\partial^{m+1} v_{j p}}{\partial x_{1}^{\left(e_{j}\right)(m+1)}}(0, t) \xi_{1}^{\left(e_{j}\right) m} \frac{\partial}{\left.\partial \xi_{1}^{\left(e_{j}\right)} \zeta\left(\frac{\xi_{1}^{\left(e_{j}\right)}}{3 r}\right)\right) \psi\left(\xi_{1}^{\left(e_{j}\right)}\right)}\right. \\
\left.+\sum_{m+p=l} \sum_{j=1}^{M}\left(\frac{1}{m !} \frac{\partial^{m} v_{j p}}{\partial x_{1}^{\left(e_{j}\right) m}}(0, t) \xi_{1}^{\left(e_{j}\right) m} \frac{\partial^{2}}{\partial \xi_{1}^{\left(e_{j}\right)}} \zeta\left(\frac{\xi_{1}^{\left(e_{j}\right)}}{3 r}\right)\right) \psi\left(\xi_{1}^{\left(e_{j}\right)}\right)\right\}
\end{gathered}
$$

where $\psi\left(\xi_{1}^{\left(e_{j}\right)}\right)=1$ if $\xi_{1}^{\left(e_{j}\right)} \geq 0$, and $\psi\left(\xi_{1}^{\left(e_{j}\right)}\right)=0$ if $\xi_{1}^{\left(e_{j}\right)}<0$; by convention, all terms depending on the local variables, vanish out of the cylinder $\Pi_{j}=\left\{\xi_{1}^{\left(e_{j}\right)} \in\right.$ $\left.(0,+\infty), \xi^{\left(e_{j}\right) \prime} \in \sigma^{\left(e_{j}\right)}\right\} ; R_{J \varepsilon}(x, t)$ is uniformly bounded by $C \varepsilon^{J-1}$, where $C$ is a constant independent of $\varepsilon$, determined by the $L^{\infty}$-norms of the derivatives $\frac{\partial^{J-l+1} v_{j p}}{\partial x_{1}^{\left(e_{j}\right)(J-l+1)}}$ and $\frac{\partial^{J-l+2} v_{j p}}{\partial x_{1}^{\left(e_{j}\right)(J-l+2)}}$.

In order to compensate these right hand sides, functions $V_{i l}^{B L}$ satisfy the equations

$$
-\Delta V_{i l}^{B L}=-F_{i l}(\xi, t)-\frac{\partial V_{i, l-2}^{B L}}{\partial t}(\xi, t),
$$

set in $\Omega_{i}=\omega_{i} \cup\left(\cup_{j: O_{i} \in e_{j}} \Pi_{j}\right)$ (here the union is taken over all $j$ such that $e_{j}$ contains $O_{i}$ as an end point), with Neumann boundary condition on $\partial \Omega_{j}$ :

$$
-\frac{\partial}{\partial n} V_{i l}^{B L}=0
$$

The existence and uniqueness of the solution to (5.6), (5.7) with exponentially decaying at infinity gradient was studied in [4]. The solution exists iff

$$
\int_{\Omega_{i}}\left\{F_{i l}(\xi, t)+\frac{\partial V_{i, l-2}^{B L}}{\partial t}(\xi, t)\right\} d \xi=0 .
$$

This condition yields:

$$
\sum_{j: O_{i} \in e_{j}} \frac{\partial v_{j l-1}}{\partial x_{1}^{\left(e_{j}\right)}}(0, t)\left|\sigma^{\left(e_{j}\right)}\right|=g_{l-1}(t)
$$


where

$$
\begin{aligned}
& g_{l}(t)= \\
& -\sum_{j: O_{i} \in e_{j}} \sum_{m+p=l, m \neq 0} \frac{1}{m !} \frac{\partial^{m+1} v_{j p}}{\partial x_{1}^{\left(e_{j}\right)(m+1)}}(0, t) \int_{\Omega_{i}} \xi_{1}^{\left(e_{j}\right) m} \frac{\partial}{\partial \xi_{1}^{\left(e_{j}\right)}} \zeta\left(\frac{\xi_{1}^{\left(e_{j}\right)}}{3 r}\right) \psi\left(\xi_{1}^{\left(e_{j}\right)}\right) d \xi \\
& (5.10) \sum_{j: O_{i} \in e_{j}} \sum_{m+p=l+1, m \geq 2} \frac{1}{m !} \frac{\partial^{m} v_{j p}}{\partial x_{1}^{\left(e_{j}\right) m}}(0, t) \int_{\Omega_{i}} \xi_{1}^{\left(e_{j}\right) m} \frac{\partial^{2}}{\partial \xi_{1}^{\left(e_{j}\right) 2}} \zeta\left(\frac{\xi_{1}^{\left(e_{j}\right)}}{3 r}\right) \psi\left(\xi_{1}^{\left(e_{j}\right)}\right) d \xi \\
& +\int_{\Omega_{i}} \frac{\partial V_{i, l-2}^{B L}}{\partial t}(\xi, t) d \xi \\
& -f\left(O_{i}, t\right) \int_{\Omega_{i}}\left\{\sum_{j: O_{i} \in e_{j}} \zeta\left(\frac{\xi_{1}^{\left(e_{j}\right)}}{3 r}\right) \psi\left(\xi_{1}^{\left(e_{j}\right)}\right)-1\right\} \chi\left(\frac{|\xi|}{e_{\min }}\right) d \xi \delta_{l 1} .
\end{aligned}
$$

This solution tends to some constants depending on time as on a parameter. Denote the constant corresponding to the outlet $\Pi_{j}$ as $c_{j l}^{i}(t)$. It is known that the solution of problem (5.6), (5.7) is unique up to an additive constant (function of $t$ ). So, we determine one of these constants, say $c_{j_{1} l}^{i}(t)=0$. Then all other constants are uniquely defined. Edge $e_{j_{1}}$ of the bundle $\mathcal{B}_{i}$ is called below the selected edge of the bundle.

These constants depend as well on the values of the derivatives of $v_{j p}$ at $(0, t)$ with $p<l$, and so these values are known from the previous steps of induction.

Let us choose now the values of $v_{j l}$ at the nodes and vertices in such a way that all constants $c_{j l}(t)$ vanish. To this end we organize the calculus of $v_{j l}$ and $V_{j l}^{B L}$ by induction in the following way.

For $l=0$ we first solve the problem on the graph $\mathcal{B}$ :

$$
\begin{gathered}
\frac{\partial v_{j 0}\left(\left(x_{1}^{\left(e_{j}\right)}, t\right)\right.}{\partial t}-\frac{\partial^{2} v_{j 0}\left(x_{1}^{\left(e_{j}\right)}, t\right)}{\partial x_{1}^{\left(e_{j}\right) 2}}=f_{j}\left(x_{1}^{\left(e_{j}\right)}, t\right), \quad x_{1}^{\left(e_{j}\right)} \in\left(0,\left|e_{j}\right|\right), t>0 \\
\sum_{j: O_{i} \in e_{j}} \frac{\partial v_{j 0}}{\partial x_{1}^{\left(e_{j}\right)}}(0, t)\left|\sigma^{\left(e_{j}\right)}\right|=0
\end{gathered}
$$

$v_{j l}(0, t)=v_{j_{1} l}(0, t), j: O_{i} \in e_{j}, j_{1}$ is the selected edge of $\mathcal{B}_{i}, i=1, \ldots, N_{1}$,

$$
\begin{gathered}
v_{j 0}(0, t)=0, i=N_{1}+1, \ldots, N, \\
v_{j 0}\left(\left(x_{1}^{\left(e_{j}\right)}, 0\right)=0,\right.
\end{gathered}
$$

and define

$$
V_{i 0}^{B L}(\xi, t)=\left\{1-\sum_{j: O_{i} \in e_{j}} \zeta\left(\frac{\xi_{1}^{\left(e_{j}\right)}}{3 r}\right) \psi\left(\xi_{1}^{\left(e_{j}\right)}\right)\right\} v_{j_{1} 0}(0, t), i=1, \ldots, N_{1}
$$

where $e_{j_{1}}$ is the selected edge of the bundle.

Assume that we have constructed $v_{j s}$ for all $s \leq l-1$, and $V_{i s}^{B L}(\xi, t), s \leq l-1$. Consider problems (5.6), (5.7) where the expressions $F_{j l}$ are defined by formulas (5.5) without the term corresponding to $m=0$ in the last sum. If we denote $\Phi_{j l}$ these new functions in the right hand sides, then 


$$
F_{i l}=\Phi_{i l}-\sum_{j: O_{i} \in e_{j}} v_{j q}(0, t) \frac{\partial^{2}}{\partial \xi_{1}^{\left(e_{j}\right) 2}} \zeta\left(\frac{x_{1}^{\left(e_{j}\right)}}{3 r \varepsilon}\right) \psi\left(\xi_{1}^{\left(e_{j}\right)}\right)
$$

Let us solve problems (5.6), (5.7) with $\Phi_{i l}$ instead of $F_{i l}$ in the right hand side. Denote $\tilde{V}_{i l}^{B L}$ its solutions. Denote $\tilde{c}_{j l}^{i}(t)$ the limits of solutions $\tilde{V}_{i l}^{B L}$ at the outlets corresponding to $\Pi_{j}$. Then consider the following problem on the graph:

$$
\begin{gathered}
\frac{\partial v_{j l}\left(\left(x_{1}^{\left(e_{j}\right)}, t\right)\right.}{\partial t}-\frac{\partial^{2} v_{j l}\left(x_{1}^{\left(e_{j}\right)}, t\right)}{\partial x_{1}^{\left(e_{j}\right) 2}}=0, \quad x_{1}^{\left(e_{j}\right)} \in\left(0,\left|e_{j}\right|\right), t>0, \\
\sum_{j: O_{i} \in e_{j}} \frac{\partial v_{j l}}{\partial x_{1}^{\left(e_{j}\right)}}(0, t)\left|\sigma^{\left(e_{j}\right)}\right|=g_{l}(t),
\end{gathered}
$$

$v_{j l}(0, t)=v_{j_{1} l}(0, t)+\tilde{c}_{j l}^{i}(t), j: O_{i} \in e_{j}, j_{1}$ is the selected edge of $\mathcal{B}_{i}, i=1, \ldots, N_{1}$,

$$
v_{j l}(0, t)=\tilde{c}_{j l}^{i}(t), i=N_{1}+1, \ldots, N
$$

$$
v_{j l}\left(\left(x_{1}^{\left(e_{j}\right)}, 0\right)=0\right.
$$

and define

$$
\begin{gathered}
V_{i l}^{B L}(\xi, t)=\tilde{V}_{i l}^{B L}(\xi, t)+\left\{1-\sum_{j: O_{i} \in e_{j}} \zeta\left(\frac{\xi_{1}^{\left(e_{j}\right)}}{3 r}\right) \psi\left(\xi_{1}^{\left(e_{j}\right)}\right)\right\} v_{j_{1} l}(0, t)- \\
\left.-\sum_{j: O_{i} \in e_{j}, j \neq j_{1}} \zeta\left(\frac{\xi_{1}^{\left(e_{j}\right)}}{3 r}\right) \psi\left(\xi_{1}^{\left(e_{j}\right)}\right)\right\} \tilde{c}_{j l}^{i}(t), i=1, \ldots, N_{1} .
\end{gathered}
$$

Note that condition (5.8) is satisfied because $v_{j, l-1}$ satisfy (5.9), see (5.14) 2 .

Now $V_{i l}^{B L}(\xi, t) \rightarrow 0$ as $|\xi| \rightarrow+\infty$.

Let us calculate the result of substitution of (5.1) in the operator $\frac{\partial}{\partial t}-\Delta$. Taking into account (5.3), we get

$$
\left(\frac{\partial}{\partial t}-\Delta\right) u_{\varepsilon}^{(J)}=f(x, t)+R_{J \varepsilon}(x, t)+R_{J \varepsilon}^{(1)}(x, t),
$$

where as it was noted above

$$
\left\|R_{J \varepsilon}\right\|_{L^{\infty}\left(B_{\varepsilon} \times(0, T)\right)} \leq C \varepsilon^{J-1}
$$

and

$$
\begin{aligned}
& R_{J \varepsilon}^{(1)}=\varepsilon^{J-1} \sum_{i=1}^{N}\left(\frac{\partial V_{i, J-1}^{B L}}{\partial t}\left(\frac{x-O_{i}}{\varepsilon}, t\right)\left(1-\zeta\left(\frac{x-O_{i}}{e_{\min }}\right)\right)\right)+ \\
& +\varepsilon^{J} \sum_{i=1}^{N}\left(\frac{\partial V_{i, J}^{B L}}{\partial t}\left(\frac{x-O_{i}}{\varepsilon}, t\right)\left(1-\zeta\left(\frac{x-O_{i}}{e_{\min }}\right)\right)\right)+R_{J \varepsilon}^{(2)}
\end{aligned}
$$


$R_{J \varepsilon}^{(2)}=\sum_{i=1}^{N}\left(\frac{\partial}{\partial t}-\Delta\right)\left(V_{i}^{B L}\left(\frac{x-O_{i}}{\varepsilon}, t\right)\left(1-\zeta\left(\frac{x-O_{i}}{e_{\min }}\right)\right)\right) \tilde{\chi}\left(\frac{x-O_{i}}{e_{\min }}\right)$,

where $\widetilde{\chi}(y)=1$ if $|y| \in\left[\frac{1}{3}, \frac{2}{3}\right], \widetilde{\chi}(y)=0$ if not.

The support of $R_{J \varepsilon}^{(2)}$ is situated in the middle third of every cylinder $B_{j \varepsilon}$, where functions $V_{i}^{B L}$ as well as their derivatives $\frac{\partial}{\partial t}, \nabla, \nabla^{2}$ are exponentially small in $L^{\infty}$-norm (see [4],[1]).

So, for $R_{J \varepsilon}^{(2)}$ (and so for $R_{J \varepsilon}^{(1)}$ as well) we get

$$
\left\|R_{J \varepsilon}^{(2)}\right\|_{L^{\infty}\left(B_{\varepsilon} \times(0, T)\right)} \leq C \varepsilon^{J-1}
$$

and

$$
\left\|R_{J \varepsilon}^{(1)}\right\|_{L^{\infty}\left(B_{\varepsilon} \times(0, T)\right)} \leq C \varepsilon^{J-1} .
$$

Here $C$ is a constant independent of $\varepsilon$.

Note that the boundary and initial conditions are satisfied by $u_{\varepsilon}^{(J)}$ exactly.

Applying now the a priori estimate (4.6), we get

$$
\left\|u_{\varepsilon}^{(J)}-u_{\varepsilon}\right\|_{H^{1}\left(B_{\varepsilon} \times(0, T)\right)} \leq C \varepsilon^{J-1}
$$

and so,

$$
\left\|u_{\varepsilon}^{(J+1)}-u_{\varepsilon}\right\|_{H^{1}\left(B_{\varepsilon} \times(0, T)\right)} \leq C \varepsilon^{J} .
$$

Comparing $u_{\varepsilon}^{(J)}$ and $u_{\varepsilon}^{(J+1)}$ we notice that

$$
\left\|u_{\varepsilon}^{(J+1)}-u_{\varepsilon}^{(J)}\right\|_{H^{1}\left(B_{\varepsilon} \times(0, T)\right)} \leq C \varepsilon^{J}
$$

with $C$ independent of $\varepsilon$. So, from the triangle inequality we get

$$
\left\|u_{\varepsilon}^{(J)}-u_{\varepsilon}\right\|_{H^{1}\left(B_{\varepsilon} \times(0, T)\right)} \leq C \varepsilon^{J} .
$$

REMARK 2. The asymptotic expansion (5.1) can be slightly modified without loss of the accuracy. Namely, the argument $\frac{\left|x-O_{i}\right|}{e_{\min }}$ in the cut off function $\zeta$ may be replaced by $C_{J} \frac{|\ln \varepsilon|\left|x-O_{i}\right|}{e_{\min }}$ where the constant $C_{J}$ is chosen in such a way that the absolute values of the boundary layer functions, as well as of their derivatives, are smaller than $\varepsilon^{J+2}$ in the zone where the cut off function is different from one and zero.

Indeed, the boundary layer functions $V_{i l}^{B L}$ and their derivatives decay exponentially: there exist positive constants $c_{1}, c_{2}$ such that

$$
\left|V_{i l}^{B L}(\xi, t)\right|,\left|\nabla^{\alpha} V_{i l}^{B L}(\xi, t)\right| \leq c_{1} \exp \left(-c_{2}|\xi|\right), \alpha=1,2,3,
$$




$$
\begin{gathered}
\left|\frac{\partial}{\partial t} V_{i l}^{B L}(\xi, t)\right|,\left|\nabla^{\alpha} \frac{\partial}{\partial t} V_{i l}^{B L}(\xi, t)\right| \leq c_{1} \exp \left(-c_{2}|\xi|\right), \alpha=1,2,3, \\
\left|\frac{\partial^{2}}{\partial t^{2}} V_{i l}^{B L}(\xi, t)\right|,\left|\nabla^{\alpha} \frac{\partial^{2}}{\partial t^{2}} V_{i l}^{B L}(\xi, t)\right| \leq c_{1} \exp \left(-c_{2}|\xi|\right), \alpha=1,2,3, \\
\left|\frac{\partial^{3}}{\partial t^{3}} V_{i l}^{B L}(\xi, t)\right|,\left|\nabla^{\alpha} \frac{\partial^{3}}{\partial t^{3}} V_{i l}^{B L}(\xi, t)\right| \leq c_{1} \exp \left(-c_{2}|\xi|\right), \alpha=1,2,3 .
\end{gathered}
$$

It follows from [4] and from the ADN-ellipticity [1] of the elliptic equations.

Therefore if $\left|x-O_{i}\right| \geq C_{J} \varepsilon|\ln \varepsilon| e_{\min } / 3$ then

$$
\begin{gathered}
\left|V_{i l}^{B L}\left(\frac{x-O_{i}}{\varepsilon}, t\right)\right| \leq c_{1} \exp \left\{-c_{2} C_{J}|\ln \varepsilon| e_{m i n} / 3\right\} \\
=c_{1} \varepsilon^{c_{2} C_{J} e_{m i n} / 3} .
\end{gathered}
$$

Choose $C_{J}$ such that

$$
c_{2} C_{J} e_{\min } / 3 \geq J+2 .
$$

Then for $V_{i l}^{B L}$ and its derivatives we get the estimate $c_{1} \varepsilon^{J+2}$. So, the difference between

$$
\zeta\left(\frac{\left|x-O_{i}\right|}{e_{\min }}\right) V_{i l}^{B L}\left(\frac{x-O_{i}}{\varepsilon}, t\right)
$$

and

$$
\zeta\left(\frac{|\ln \varepsilon|\left|x-O_{i}\right|}{e_{\min }}\right) V_{i l}^{B L}\left(\frac{x-O_{i}}{\varepsilon}, t\right)
$$

can be estimated by

$$
\left|V_{i l}^{B L}\left(\frac{x-O_{i}}{\varepsilon}, t\right)\right| \leq c_{1} \varepsilon^{J+2}
$$

in the domain

$$
\operatorname{supp}\left\{\zeta\left(\frac{\left|x-O_{i}\right|}{e_{\min }}\right)-\zeta\left(\frac{|\ln \varepsilon|\left|x-O_{i}\right|}{e_{\min }}\right)\right\}
$$

where $\frac{\left|x-O_{i}\right|}{C_{J}|\ln \varepsilon| e_{\min }} \geq 1 / 3$.

In the same way we get the similar estimate for the derivatives of this difference. It means that the change of the argument $\frac{\left|x-O_{i}\right|}{e_{\min }}$ by $\frac{|\ln \varepsilon|\left|x-O_{i}\right|}{C_{J} e_{\min }}$ in $\zeta$ gives an 
additional residual of order $\varepsilon^{J}$ (the factor $\varepsilon^{-2}$ appears after two derivations in $x$ variable), and so does not lead to any loss of the accuracy.

Denote $u_{a \varepsilon}^{(J)}$ so modified expansion (5.1). So,

$$
\left\|u_{a \varepsilon}^{(J)}-u_{\varepsilon}\right\|_{H^{1}\left(B_{\varepsilon} \times(0, T)\right)} \leq C \varepsilon^{J} .
$$

REMARK 3. In the same way as above one can calculate the partial derivatives of the residual $R_{J}=R_{J \varepsilon}(x, t)+R_{J \varepsilon}^{(1)}(x, t)$ and get the following estimate:

$$
\left\|R_{J}\right\|_{W^{\beta, \infty}\left(B_{\varepsilon} \times(0, T)\right)} \leq C \varepsilon^{J-1-\beta}, \beta=1,2,
$$

so that for the residual $R_{J+1}$ (result of substitution of $u_{a \varepsilon}^{(J+1)}$ into the heat equation) satisfies

$$
\left\|R_{J+1}\right\|_{W^{\beta, \infty}\left(B_{\varepsilon} \times(0, T)\right)} \leq C \varepsilon^{J-\beta}, \beta=1,2 .
$$

6. Asymptotic partial decomposition of the domain for the heat equation. In this section we apply the method of partial asymptotic decomposition of the domain assuming that $f_{j}$ are $C^{J+4}$-smooth functions, defined in Section 4 .

Let us describe the algorithm of the MAPDD for the heat equation set in a tube structure $B_{\varepsilon}$. Let $\delta$ be a small positive number much greater than $\varepsilon$ (it will be chosen of order $O(\varepsilon|\ln \varepsilon|))$. For any edge $e=\left[O_{i}, O_{j}\right]$ connecting two nodes of the graph of the structure introduce two hyperplanes orthogonal to this edge and crossing it at the distance $\delta$ from its ends. Denote the cross-sections of the cylinder $B_{\varepsilon}^{(e)}$ containing $e$ by these two hyperplanes respectively, $S_{i, j}$ (at the distance $\delta$ from $O_{i}$ ), and $S_{j, i}$ (at the distance $\delta$ from $O_{j}$ ), and denote the part of the cylinder $B_{\varepsilon}^{(e)}$ between these two cross-sections by $B_{i j}^{\text {dec, } \varepsilon}$. Denote $B_{i}^{\varepsilon, \delta}$ the connected truncated by the cross sections $S_{i, j}$, part of $B_{\varepsilon}$ containing the vertex or the node $O_{i}$. Denote $e_{i j}^{d e c, \delta}$ the part of the edge $\left[O_{i}, O_{j}\right]$ concluded between the cross-sections $S_{i, j}$ and $S_{j, i}$.

If $O_{i}$ is a node and $O_{j}$ is a vertex, then we trace only one hyperplan $S_{i, j}$ (at the distance $\delta$ from $O_{i}$ ), and denote the part of the cylinder $B_{\varepsilon}^{(e)}$ between $S_{i, j}$ and the cylinder's base $\gamma_{\varepsilon}^{j}$ by $B_{i j}^{d e c, \varepsilon}$. Denote $e_{i j}^{d e c, \delta}$ the part of the edge $\left[O_{i}, O_{j}\right]$ concluded between $S_{i, j}$ and $\gamma_{\varepsilon}^{j}$.

Define subspace $H_{\gamma, 0}^{1}\left(B_{\varepsilon} \times(0, T), \delta\right)\left(H_{\gamma, 0}^{1}\left(B_{\varepsilon}, \delta\right)\right)$ of the space $H_{\gamma, 0}^{1}\left(B_{\varepsilon} \times(0, T)\right)$ (respectively, $H_{\gamma, 0}^{1}\left(B_{\varepsilon}\right)$, such that its elements have vanishing transversal derivatives $\nabla_{x(e)}^{\prime}$ on every truncated cylinder $B_{i j}^{d e c, \varepsilon}$.

The method of asymptotic partial domain decomposition (MAPDD) replaces the problem (4.1) by its projection on $H_{\gamma, 0}^{1}\left(B_{\varepsilon} \times(0, T), \delta\right)$ : find $u_{\varepsilon, \delta, d e c} \in H_{\gamma, 0}^{1}\left(B_{\varepsilon} \times\right.$ $(0, T), \delta)$ such that for almost all $t \in(0, T)$,

$$
\int_{B_{\varepsilon}}\left(\frac{\partial u_{\varepsilon, \delta, d e c}}{\partial t} v+\nabla u_{\varepsilon, \delta, d e c} \cdot \nabla v\right) d x=\int_{B_{\varepsilon}} f v d x, \quad v \in H_{\gamma, 0}^{1}\left(B_{\varepsilon}, \delta\right),
$$

and satisfying

$$
\left.u_{\varepsilon, \delta, \operatorname{dec}}\right|_{t=0}=0,
$$


which implies:

$\int_{B_{\varepsilon} \times(0, T)}\left(\frac{\partial u_{\varepsilon, \delta, d e c}}{\partial t} v+\nabla u_{\varepsilon, \delta, d e c} \cdot \nabla v\right) d x d t=\int_{B_{\varepsilon} \times(0, T)} f v d x d t, \quad v \in H_{\gamma, 0}^{1,0}\left(B_{\varepsilon} \times(0, T), \delta\right)$,

$$
\left.u_{\varepsilon, \delta, d e c}\right|_{t=0}=0 \text {. }
$$

where

$$
H_{\gamma, 0}^{1,0}\left(B_{\varepsilon} \times(0, T), \delta\right)=\left\{v \in H_{\gamma, 0}^{1,0}\left(B_{\varepsilon} \times(0, T)\right) ; \nabla_{x^{(e)}}^{\prime} v=0\right\} .
$$

THEOREM 6.1. There exists a unique solution of this partially decomposed problem. The estimate holds

$$
\left\|u_{\varepsilon, \delta, d e c}\right\|_{H^{1}\left(B_{\varepsilon} \times(0, T)\right)} \leq C_{P F}\|f\|_{L^{2}\left(B_{\varepsilon} \times(0, T)\right)}
$$

where the constant $C_{P F}$ is independent of $\varepsilon$ and $\delta$.

The proof of this theorem repeats the proof of Theorem 4.1, where the Galerkin base is constructed in the space $H_{\gamma, 0}^{1}\left(B_{\varepsilon}, \delta\right)$ instead of $H_{\gamma, 0}^{1}\left(B_{\varepsilon}\right)$.

REMARK 4. This estimate (6.5) holds in the case if the right hand side is any function of $L^{2}\left(B_{\varepsilon} \times(0, T)\right)$ free of the above regularity restrictions (and so it can depend on all components of $x)$.

THEOREM 6.2. Let $\delta$ satisfy the following inequality

$$
\delta \geq C_{J+1} \varepsilon|\ln (\varepsilon)|
$$

where $C_{J+1}$ is chosen according to (5.18). Then the estimate holds for the difference $u_{\varepsilon}-u_{\varepsilon, \delta, d e c}$ :

$$
\left\|u_{\varepsilon}-u_{\varepsilon, \delta, \operatorname{dec}}\right\|_{H^{1}\left(B_{\varepsilon} \times(0, T)\right)} \leq C \varepsilon^{J},
$$

where constant $C$ is independent of $\varepsilon$.

This estimate justifies the method of asymptotic partial decomposition of domain for the heat equation. Its proof is given in [7].

Notice that the integration by parts in the variational formulation (6.1) gives the differential version of the partially decomposed problem. Namely, denoting $\hat{u}$ the restriction of $u$ on the part $e_{i j}^{d e c, \delta}$ of the edge $e$ we have

$$
\begin{gathered}
\frac{\partial u_{\varepsilon, \delta, d e c}}{\partial t}-\Delta u_{\varepsilon, \delta, d e c}=f(x, t), \quad x \in B_{i}^{\varepsilon, \delta}, i=1, \ldots, N_{1}, t \in(0, T), \\
\frac{\partial \hat{u}_{\varepsilon, \delta, d e c}}{\partial t}-\frac{\partial^{2} \hat{u}_{\varepsilon, \delta, d e c}}{\partial x_{1}^{(e) 2}}=\hat{f}\left(x_{1}^{(e)}, t\right), \quad x \in e_{i j}^{d e c, \delta}, \forall e ; t \in(0, T) \\
\frac{\partial u_{\varepsilon, \delta, d e c}}{\partial n}=0, \quad x \in\left(\partial B_{i}^{\varepsilon, \delta} \cap \partial B^{\varepsilon}\right) \backslash \gamma_{\varepsilon}, i=1, \ldots, N_{1}, t \in(0, T) \\
u_{\varepsilon, \delta, d e c}=0, \quad x \in \gamma_{\varepsilon}, t \in(0, T) \\
u_{\varepsilon, \delta, d e c}(x, 0)=0, x \in B_{\varepsilon} .
\end{gathered}
$$

with the junction condition at the sections $S_{i j}$ corresponding to the value $x_{1}^{(e)}=\delta$ for the local variable, which are the same as in [5]: 


$$
\begin{aligned}
& \left.u_{\varepsilon, \delta, \operatorname{dec}}(x, t)\right|_{x_{1}^{(e)}=\delta}=\hat{u}_{\varepsilon, \delta, \operatorname{dec}}(\delta, t), \\
& \left.\frac{1}{\left|S_{i j}\right|} \int_{S_{i j}} \frac{\partial u_{\varepsilon, \delta, d e c}}{\partial x_{1}^{(e)}} d x^{(e) \prime}\right|_{x_{1}^{(e)}=\delta}=\frac{\partial \hat{u}_{\varepsilon, \delta, d e c}}{\partial x_{1}^{(e)}}(\delta, t) .
\end{aligned}
$$

Here, $\hat{f}\left(x_{1}^{(e)}(x), t\right)=f(x, t)$, for $x \in e_{i j}^{d e c, \delta}$.

It means that we keep the $n$-dimensional in space setting $(6.8)_{1}$ for the heat equation within small pieces $B_{i}^{\varepsilon, \delta}, i=1, \ldots, N$, (their diameters are of order $\varepsilon|\ln (\varepsilon)|$ ), reduce the dimension to one and consider the heat equation $(6.8)_{2}$ on the pieces $e_{i j}^{\text {dec, } \delta}$ of edges $e$ and add the junction conditions (6.9) between the $n$-dimensional and one dimensional parts. This reduction allows us to reduce the mesh $\frac{1}{\varepsilon|\ln (\varepsilon)|}$ times and to keep an exponential precision of the computations.

Note that conditions (6.9) are "dissipative" in the following sense. Assume that the right hand side $f$ vanishes for all $t \in\left[t_{1}, t_{2}\right], \quad t_{1}<t_{2}$. Then (6.3) with $v=u_{\varepsilon, \delta, d e c}$ yields:

$$
\int_{B_{\varepsilon}} u_{\varepsilon, \delta, d e c}^{2}\left(x, t_{2}\right) d x \leq \int_{B_{\varepsilon}} u_{\varepsilon, \delta, d e c}^{2}\left(x, t_{1}\right) d x .
$$

THEOREM 6.3. Assume that $f_{j}$ are $C^{J+5}$-smooth functions with a compact support vanishing in some independent of $\varepsilon$ neighborhood of the nodes. Let $\delta$ satisfy the following inequality

$$
\delta \geq C_{J+1} \varepsilon|\ln (\varepsilon)|
$$

where $C_{J+1}$ is chosen according to (5.18). Then the following estimate holds :

$$
\begin{gathered}
\left\|\nabla^{2}\left(u_{a \varepsilon}^{(J+1)}-u_{\varepsilon, \delta, d e c}\right)\right\|_{L^{\infty}\left(B_{\varepsilon} \times(0, T)\right)} \leq C \varepsilon^{J-5}, \\
\left\|u_{a \varepsilon}^{(J+1)}-u_{\varepsilon, \delta, d e c}\right\|_{W^{1, \infty}\left(B_{\varepsilon} \times(0, T)\right)} \leq C \varepsilon^{J-5},
\end{gathered}
$$

For all $J \geq 5$,

$$
\left\|\nabla u_{\varepsilon, \delta, \operatorname{dec}}\right\|_{L^{\infty}\left(B_{\varepsilon} \times(0, T)\right)} \leq C, \quad\left\|\nabla^{2} u_{\varepsilon, \delta, \operatorname{dec}}\right\|_{L^{\infty}\left(B_{\varepsilon} \times(0, T)\right)} \leq C \varepsilon^{-1},
$$

where constant $C$ is independent of $\varepsilon$.

Proof. Let $w=u_{a \varepsilon}^{(J+1)}-u_{\varepsilon, \delta, d e c}$; it belongs to $H_{\gamma, 0}^{1}\left(B_{\varepsilon} \times(0, T), \delta\right)$ and satisfies the equation

$$
\begin{gathered}
\frac{\partial w}{\partial t}-\triangle w=R_{J+1}, x \in B_{\varepsilon} \backslash\left(\cup_{i=1}^{N_{1}} \cup_{j=1}^{N} S_{i j}\right) \\
R_{J+1}=R_{J+1, \varepsilon}+R_{J+1, \varepsilon}^{(1)} .
\end{gathered}
$$

At the interfaces $S_{i j}, w$ is a constant with respect to $x$ and the integral flux conservation (6.9) holds (for $w$ ). $R_{J+1}=0$ on $S_{i j}$.

Deriving (6.12) in time, we can write

$$
\frac{\partial^{\alpha+1} w}{\partial t^{\alpha+1}}-\triangle \frac{\partial^{\alpha} w}{\partial t^{\alpha}}=\frac{\partial^{\alpha} R_{J+1}}{\partial t^{\alpha}}
$$


$\alpha=1,2,3,4$. So we can write the following estimates :

1)

$$
\left\|\frac{\partial^{4} w}{\partial t^{4}}\right\|_{L^{2}\left(B_{\varepsilon} \times(0, T)\right)} \leq C\left\|\frac{\partial^{3} R_{J+1}}{\partial t^{3}}\right\|_{L^{2}\left(B_{\varepsilon} \times(0, T)\right)}
$$

(This estimate follows from the standard a priori estimate for the equation

$$
\left.\frac{\partial^{4} w}{\partial t^{4}}-\triangle \frac{\partial^{3} w}{\partial t^{3}}=\frac{\partial^{3} R_{J+1}}{\partial t^{3}}\right)
$$

2)

$$
\begin{aligned}
& \left\|\frac{\partial^{3} w}{\partial t^{3}}\right\|_{L^{2}\left(0, T ; H^{2}\left(B_{\varepsilon}\right)\right.} \leq C\left(\left\|\frac{\partial^{3} w}{\partial t^{3}}\right\|_{L^{2}\left(B_{\varepsilon} \times(0, T)\right)}\right. \\
+\left\|\frac{\partial^{4} w}{\partial t^{4}}\right\|_{L^{2}\left(B_{\varepsilon} \times(0, T)\right)} & \left.+\left\|\frac{\partial^{3} R_{J+1}}{\partial t^{3}}\right\|_{L^{2}\left(B_{\varepsilon} \times(0, T)\right)}\right)
\end{aligned}
$$

(This estimate follows from the ADN (S. Agmon, A. Douglis, L. Nirenberg) theory [1] for the equation

$$
-\triangle \frac{\partial^{3} w}{\partial t^{3}}=\frac{\partial^{3} R_{J+1}}{\partial t^{3}}-\frac{\partial^{4} w}{\partial t^{4}}
$$

with $\frac{\partial^{4} w}{\partial t^{4}}$ transposed to the right hand side. To this end we scale the equation and the domain passing to the variables $\xi=\frac{x}{\varepsilon}$, apply the ADN theory and then make the back change $x=\varepsilon \xi)$.

3)

$$
\begin{aligned}
& \left\|\frac{\partial^{2} w}{\partial t^{2}}\right\|_{L^{2}\left(0, T ; H^{4}\left(B_{\varepsilon}\right)\right.} \leq C \varepsilon^{-2}\left(\left\|\frac{\partial^{2} w}{\partial t^{2}}\right\|_{L^{2}\left(0, T ; L^{2}\left(B_{\varepsilon}\right)\right)}\right) \\
& \left.+\left\|\frac{\partial^{4} w}{\partial t^{4}}\right\|_{L^{2}\left(0, T ; H^{2}\left(B_{\varepsilon}\right)\right.}+\left\|\frac{\partial^{2} R_{J+1}}{\partial t^{2}}\right\|_{L^{2}\left(0, T ; H^{2}\left(B_{\varepsilon}\right)\right.}\right)
\end{aligned}
$$

(This estimate follows from the ADN theory for the elliptic equation

$$
-\triangle \frac{\partial^{2} w}{\partial t^{2}}=\frac{\partial^{2} R_{J+1}}{\partial t^{2}}-\frac{\partial^{3} w}{\partial t^{3}}
$$

The factor $\varepsilon^{-2}$ appears because after the back change, the fourth derivatives of $\frac{\partial^{2} w}{\partial t^{2}}$ in space will give the factor $\varepsilon^{4}$.)

Another possibility to prove the estimate is to use the result [16].

REMARK 5. At the interfaces $S_{i j}$, we cannot apply the ADN theory directly because of the angles at the boundary of every component $B_{i}^{\varepsilon, \delta}$. However, this obstacle is removed by an "odd" extension of the problem through the truncating plane $S_{i j}$. Indeed, the value of the function $w$ on this cross section is a function depending only on time : $w=U(t)$. Consider the domain $\widetilde{B}_{i}^{\varepsilon, \delta}=B_{i}^{\varepsilon, \delta} \cup S B^{\varepsilon, \delta}$, where $S B^{\varepsilon, \delta}$ is the symmetric reflection of $B_{i}^{\varepsilon, \delta}$ with respect to the plane $S_{i j}$. We assume that $\varepsilon$ is small enough for non-overlapping of $B_{i}^{\varepsilon, \delta}$ and its symmetric reflection. Extend the 
heat equation (or its time derivative) from $B_{i}^{\varepsilon, \delta}$ to $\widetilde{B}_{i}^{\varepsilon, \delta}$ in a way that $w-U(t)$ is odd with respect $S_{i j}$ as it is done in [10]. Then the above ADN estimates can be written everywhere in $\widetilde{B}_{i}^{\varepsilon, \delta}$, and in particular, at $S_{i j}$. Similarly, the problem of angles at the bases $\gamma_{\varepsilon}$ of the cylinders is removed due to the possibility of an odd extension of the heat equation through these bases $\gamma_{\varepsilon}$. Remind that the right hand side is equal to zero in the neighborhood of the bases and that for small values of $t\left(t \leq t_{0}\right), u_{\varepsilon, \delta, d e c}=0$.

(6.14)-(6.16) yield:

$$
\left\|\frac{\partial^{2} w}{\partial t^{2}}\right\|_{L^{2}\left(0, T ; H^{4}\left(B_{\varepsilon}\right)\right)} \leq C \varepsilon^{J-3}
$$

and so,

$$
\|w\|_{H^{2}\left(0, T ; H^{4}\left(B_{\varepsilon}\right)\right)} \leq C \varepsilon^{J-3}
$$

So,

$$
\|\nabla w\|_{H^{2}\left(B_{\varepsilon} \times(0, T)\right)} \leq C \varepsilon^{J-3} \text { and }\left\|\nabla w^{2}\right\|_{H^{2}\left(B_{\varepsilon} \times(0, T)\right)} \leq C \varepsilon^{J-3}
$$

Applying now the Sobolev imbedding theorem to the finite number of parts of $B_{\varepsilon}$, we get:

$$
\begin{gathered}
\|\nabla w\|_{L^{\infty}\left(B_{\varepsilon} \times(0, T)\right)} \leq C \varepsilon^{-2}\|\nabla w\|_{H^{2}\left(B_{\varepsilon} \times(0, T)\right)} \\
\left\|\nabla^{2} w\right\|_{L^{\infty}\left(B_{\varepsilon} \times(0, T)\right)} \leq C \varepsilon^{-2}\left\|\nabla^{2} w\right\|_{H^{2}\left(B_{\varepsilon} \times(0, T)\right)}
\end{gathered}
$$

i.e.

$$
\left\|\nabla^{\beta} w\right\|_{L^{\infty}\left(B_{\varepsilon} \times(0, T)\right)} \leq C \varepsilon^{J-5}, \beta=1,2
$$

In the same way differentiating $(6.12)$ in times we get the estimate for $\frac{\partial w}{\partial t}$ :

$$
\left\|\frac{\partial w}{\partial t}\right\|_{L^{\infty}\left(B_{\varepsilon} \times(0, T)\right)} \leq C \varepsilon^{J-5}
$$

Calculating the norms

$$
\left\|\nabla_{(x, t)}^{\beta} u_{a \varepsilon}^{(J+1)}\right\|_{L^{\infty}\left(B_{\varepsilon} \times(0, T)\right)}
$$

for $\beta=1,2$, we find $O\left(\varepsilon^{1-\beta}\right)$, and from the previous estimate (6.10) for $J \geq 5$ we get estimate (6.11). The theorem is proved.

7. Numerical scheme. From now on, we consider the heat equation set in a simple two-dimensional $(n=2)$ rod structure containing one bundle $\left(N_{1}=1\right)$. Let $O_{1}$ be the only one node, and $O_{j}, j \in\{2, \ldots, M+1\}$ be the vertices, each $e_{j}, j \in\{1, \ldots, M\}$, being the closed segment connecting $O_{1}$ and $O_{j+1}$. Let $l_{j}=\left|e_{j}\right|$ be the length of $e_{j}$, and $\theta_{1}, \ldots, \theta_{M}$ be some positive numbers independent of $\varepsilon$. 
We define here as in Section 3

$$
\begin{aligned}
& B_{\varepsilon}^{\left(e_{j}\right)}=\left\{x^{\left(e_{j}\right)} \in \mathbb{R}^{2}: x_{1}^{\left(e_{j}\right)} \in\left(0, l_{j}\right), x_{2}^{\left(e_{j}\right)} \in\left(-\frac{\varepsilon \theta_{j}}{2}, \frac{\varepsilon \theta_{j}}{2}\right)\right\} \\
& B_{\varepsilon}=\left(\cup_{j=1}^{M} B_{\varepsilon}^{\left(e_{j}\right)}\right) \cup \omega_{\varepsilon}^{1} \\
& S_{1, j}=\left\{x^{\left(e_{j-1}\right)} \in B_{\varepsilon}^{\left(e_{j-1}\right)}: x_{1}^{\left(e_{j-1}\right)}=\delta\right\}, j \in\{2, \ldots, M+1\} \\
& B_{1}^{\varepsilon, \delta} \text { is the truncated by the cross-sections } S_{1, j}, j \in\{2, \ldots, M+1\} \text { part } \\
& \text { of } B_{\varepsilon} \text { containing } O_{1} \text {. } \\
& B_{1, j}^{d e c, \varepsilon} \text { is the part of } B_{\varepsilon}^{\left(e_{j-1}\right)} \text { between } S_{1, j} \text { and } \gamma_{\varepsilon}^{j}, j \in\{2, \ldots, M+1\} . \\
& e_{1, j}^{\text {dec, },} \text { is the part of } e_{j-1} \text { between } S_{1, j} \text { and } \gamma_{\varepsilon}^{j}, j \in\{2, \ldots, M+1\} .
\end{aligned}
$$
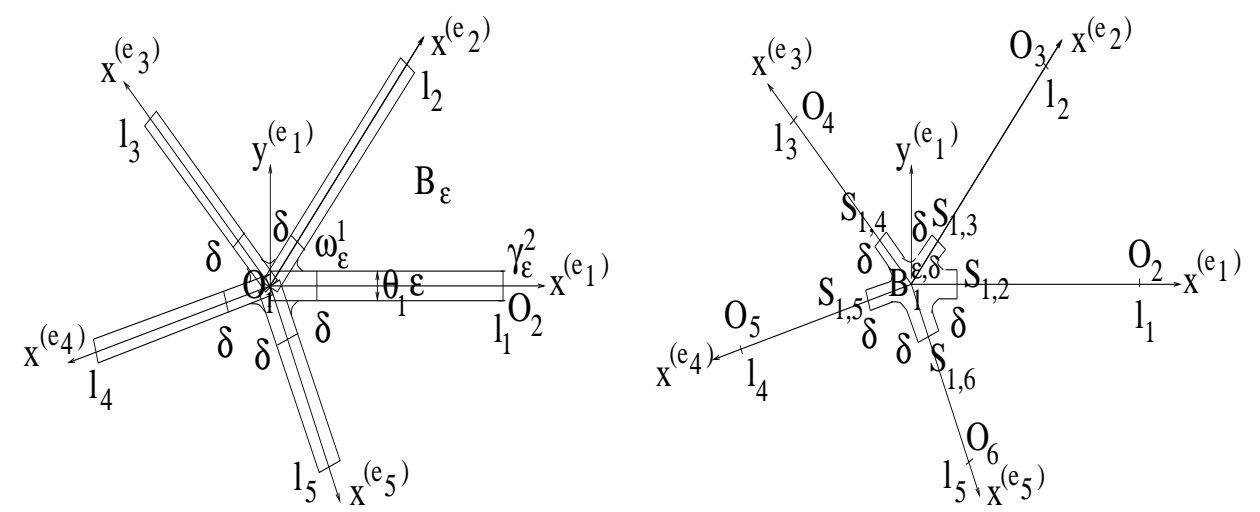

FIG. 7.1. (a) $B_{\varepsilon}$ and (b) $D_{\varepsilon}$.

Let $D_{\varepsilon}=B_{1}^{\varepsilon, \delta} \cup\left(\cup_{j=2}^{M+1} e_{1, j}^{d e c, \delta}\right)$.

The following partially decomposed problem obtained by rewriting (6.8) in $D_{\varepsilon}$ is considered.

$$
\left\{\begin{array}{l}
\frac{\partial v_{j}}{\partial t}\left(x^{\left(e_{j}\right)}, t\right)-\frac{\partial^{2} v_{j}}{\partial x_{1}^{\left(e_{j}\right) 2}}\left(x^{\left(e_{j}\right)}, t\right)=f_{j}\left(x^{\left(e_{j}\right)}, t\right), x_{1}^{\left(e_{j}\right)} \in\left(\delta, l_{j}\right) \\
j=1, \ldots, M, t \in(0, T) \quad(a) \\
v_{j}\left(x^{\left(e_{j}\right)}, 0\right)=0, x_{1}^{\left(e_{j}\right)} \in\left(\delta, l_{j}\right), j=1, \ldots, M \\
v_{j}\left(l_{j}, t\right)=0, t \in(0, T) \\
\frac{\partial u}{\partial t}(x, t)-\triangle u(x, t)=f(x, t), x \in B_{1}^{\varepsilon, \delta}, t \in(0, T) \quad(b) \\
u(x, 0)=0, x \in B_{1}^{\varepsilon, \delta} \\
\frac{\partial u}{\partial n}(x, t)=0, x \in \partial B_{1}^{\varepsilon, \delta} \backslash\left(\cup_{j=1}^{n} S_{1, j+1}\right), t \in(0, T) \\
u(x, t)=v_{j}(\delta, t), x \in S_{1, j+1}, j=1, \ldots, M, t \in(0, T) \\
\frac{\partial v_{j}}{\partial x_{1}^{\left(e_{j}\right)}}(\delta, t)=\frac{1}{\theta_{j} \varepsilon} \int_{S_{1, j+1}} \frac{\partial u}{\partial n} d \gamma, j=1, \ldots, M, t \in(0, T) \quad(c)
\end{array}\right.
$$

Denote $u_{\varepsilon, \delta, \operatorname{dec}}(x, t)=\left\{\begin{array}{ll}u(x, t) & \text { if } \quad x \in B_{1}^{\varepsilon, \delta} \\ v_{j}\left(x_{1}^{\left(e_{j}\right)}, t\right) & \text { if } \quad x \in B_{1, j+1}^{\text {dec, }}, j \in\{1, \ldots, M\}\end{array}, t \in(0, T)\right.$, the solution of the partially decomposed problem (7.2). Sections 7 and 8 aim to obtain a finite volume approximation $u_{\varepsilon, \delta, d e c, \mathcal{T}, k}$ for $u_{\varepsilon, \delta, d e c}$ and to get an estimate for the 
difference $u_{\varepsilon}-u_{\varepsilon, \delta, d e c, \mathcal{T}, k}$. Thanks to the estimate (6.7) of Theorem 6.2, it is sufficient to evaluate $\left\|u_{\varepsilon, \delta, d e c}-u_{\varepsilon, \delta, d e c, \mathcal{T}, k}\right\|$ in a suitable norm. In the following, the dependence of this estimate on $\varepsilon$ and the size of the meshes is highlighted.

7.1. The meshes. Let us define a mesh of $e_{1, j+1}^{d e c, \delta}$ on the axis $O_{1} x_{1}^{\left(e_{j}\right)}, j=$ $1, \ldots, M$. For each value of $j=1, \ldots, M$, we choose $N_{j} \in \mathbb{N}^{*}$, and $N_{j}+1$ distinct and increasing values $x_{i+1 / 2}^{e_{j}}, i=0, \ldots, N_{j}$, such that $x_{1 / 2}=\delta, x_{N_{j}+1 / 2}=l_{j}$. Denote $I_{i}^{e_{j}}=\left(x_{i-1 / 2}^{e_{j}}, x_{i+1 / 2}^{e_{j}}\right)$, and $h_{i}^{e_{j}}=x_{i+1 / 2}^{e_{j}}-x_{i-1 / 2}^{e_{j}}, i=1, \ldots, N_{j}$.

Set $h^{e_{j}}=\max \left(h_{i}^{e_{j}}, i=1, \ldots, N_{j}\right)$ the size of the mesh of the interval $\left(\delta, l_{j}\right)$.

Then we choose $N_{j}$ points $x_{i}^{e_{j}}, i=1, \ldots, N_{j}$, such that $x_{i}^{e_{j}} \in I_{i}^{e_{j}}$. Set $x_{0}^{e_{j}}=\delta, x_{N_{j}+1}^{e_{j}}=$ $l_{j}$, and $h_{i+1 / 2}^{e_{j}}=x_{i+1}^{e_{j}}-x_{i}^{e_{j}}, i=0, \ldots, N_{j}$.

Let us construct an admissible mesh over $B_{1}^{\varepsilon, \delta}$ denoted by $\mathcal{T}_{1}$. For the sake of simplicity, it is convenient to assume till the end of Section 7 that $B_{1}^{\varepsilon, \delta}$ is polygonal. We remind (see the definition in [18]) that such a mesh consists in a family of open polygonal convex subsets $K$ of $B_{1}^{\varepsilon, \delta}$ (with positive measures) called control volumes, a family of edges $\sigma$ (with strictly positive measures) of the control volumes denoted by $\mathcal{E}$, and a family of points $x_{K}$ chosen in each control volume $K$ denoted by $\mathcal{P}$. The mesh $\mathcal{T}_{1}$ satisfies the following properties

1) The closure of the union of all the control volumes is $\overline{B_{1}^{\varepsilon, \delta}}$.

2) For any $K \in \mathcal{T}_{1}$, there is a subset $\mathcal{E}_{K}$ of $\mathcal{E}$ such that $\partial K=\bigcup_{\sigma \in \mathcal{E}_{K}} \bar{\sigma}$, and $\bigcup_{K \in \mathcal{T}_{1}} \mathcal{E}_{K}=\mathcal{E}$.

3) For any $(K, L) \in \mathcal{T}_{1}^{2}, K \neq L$, one of three following assertions holds:

(7.3) either $\bar{K} \cap \bar{L}=\emptyset$, or $\bar{K} \cap \bar{L}$ is a common vertex of $\mathrm{K}$ and $\mathrm{L}$,

or $\bar{K} \cap \bar{L}=\bar{\sigma}, \sigma$ being a common edge of $\mathrm{K}$ and $\mathrm{L}$ denoted by $\sigma_{K / L}$.

4) The family $\mathcal{P}=\left(x_{K}\right)_{K \in \mathcal{T}_{1}}$ is such that for any $K \in \mathcal{T}_{1}, x_{K} \in \bar{K}$.

For any $(K, L) \in \mathcal{T}_{1}^{2}, K \neq L$, it is assumed that $x_{K} \neq x_{L}$ and that

the straight line going through $x_{K}$ and $x_{L}$ is orthogonal to $\sigma_{K / L}$.

5) For any $\sigma \in \mathcal{E}$, if $\sigma \subset \partial B_{1}^{\varepsilon, \delta}, \sigma \in \mathcal{E}_{K}$ and $x_{K} \notin \sigma$, the orthogonal

projection of $x_{K}$ on the straight line containing the edge $\sigma$, belongs to $\sigma$.

Let $\mathcal{E}_{\text {int }}=\left\{\sigma \in \mathcal{E}, \sigma \not \subset \partial B_{1}^{\varepsilon, \delta}\right\}$.

For any $(K, L) \in \mathcal{T}_{1}^{2}, K \neq L$, if $\sigma=\sigma_{K / L}$, let $d_{\sigma}$ be the distance between $x_{K}$ and $x_{L}$. For any $K \in \mathcal{T}_{1}$, if $\sigma \in \mathcal{E}_{K}$ and if $\sigma \subset \partial B_{1}^{\varepsilon, \delta}$, let $d_{\sigma}$ be the distance between $x_{K}$ and $\sigma$.

We assume that for any $\sigma \in \mathcal{E}, d_{\sigma} \neq 0$.

For any $K \in \mathcal{T}_{1}$, let $m(K)$ be the area of $K$. For any $\sigma \in \mathcal{E}$, let $m(\sigma)$ be the length of $\sigma$. Let $h_{0}$ be the size of the mesh $\mathcal{T}_{1}, h_{0}=\max \left\{\partial(K), K \in \mathcal{T}_{1}\right\}$, where $\partial(K)$ is the diameter of $K$.

The mesh of $D_{\varepsilon}$, which is the union of $\mathcal{T}_{1}$ and the meshes of $e_{1, j}^{d e c, \delta}, j \in\{2, \ldots, M+$ $1\}$, is denoted by $\mathcal{T}$. Let $h$ be the size of $\mathcal{T}, h=\max \left\{h_{0}, h^{e_{j}}, j=1, \ldots, M\right\}$.

7.2. The hybrid scheme. A constant time step $k \in(0, T)$ is merely chosen for the time discretization. Let $N_{k} \in \mathbb{N}^{*}$ such that $N_{k}=\max \{n \in \mathbb{N}, n k<T\}$. Let $t_{n}=$ $n k$, for $n \in\left\{0, \ldots, N_{k}+1\right\}$. The scheme is obtained by integrating $\frac{\partial v_{j}}{\partial t}-\frac{\partial^{2} v_{j}}{\partial x_{1}^{\left(e_{j}\right) 2}}=f_{j}$ 
on each cell $I_{i}^{e_{j}}, i=1, \ldots, N_{j}$, and $\frac{\partial u}{\partial t}-\triangle u=0$ over each control volume $K \in \mathcal{T}_{1}$, and time interval $(n k,(n+1) k)$, for $n \in\left\{0, \ldots, N_{k}\right\}, j \in\{1, \ldots, M\}$. The numerical flux $F_{i+1 / 2}^{j, n+1}$ is an approximation of $-\frac{\partial v_{j}}{\partial x_{1}^{\left(e_{j}\right)}}\left(x_{i+1 / 2}^{e_{j}}, t\right), t \in(n k,(n+1) k)$ of finite difference type; the value $v_{j, i}^{n}$ is an approximation of $v_{j}\left(x_{i}^{e_{j}}, t_{n}\right), i=0, \ldots, N_{j}+1$. The flux - $\int_{\sigma} \nabla u . n$ through the edge $\sigma$ of the cell $K \in \mathcal{T}_{1}$ on $(n k,(n+1) k)$ is approximated by a differential quotient we term $F_{K, \sigma}^{n+1}$; the value $u_{K}^{n}$ is an approximation of $u\left(x_{K}, t_{n}\right), K \in$ $\mathcal{T}_{1}, n \in\left\{0, \ldots, N_{k}+1\right\}$. Since the stability condition for an explicit discretization is too strong a condition in terms of computational cost, an implicit formulation is chosen.

The hybrid scheme below is proposed to approach the solution of the partially decomposed problem (7.2):

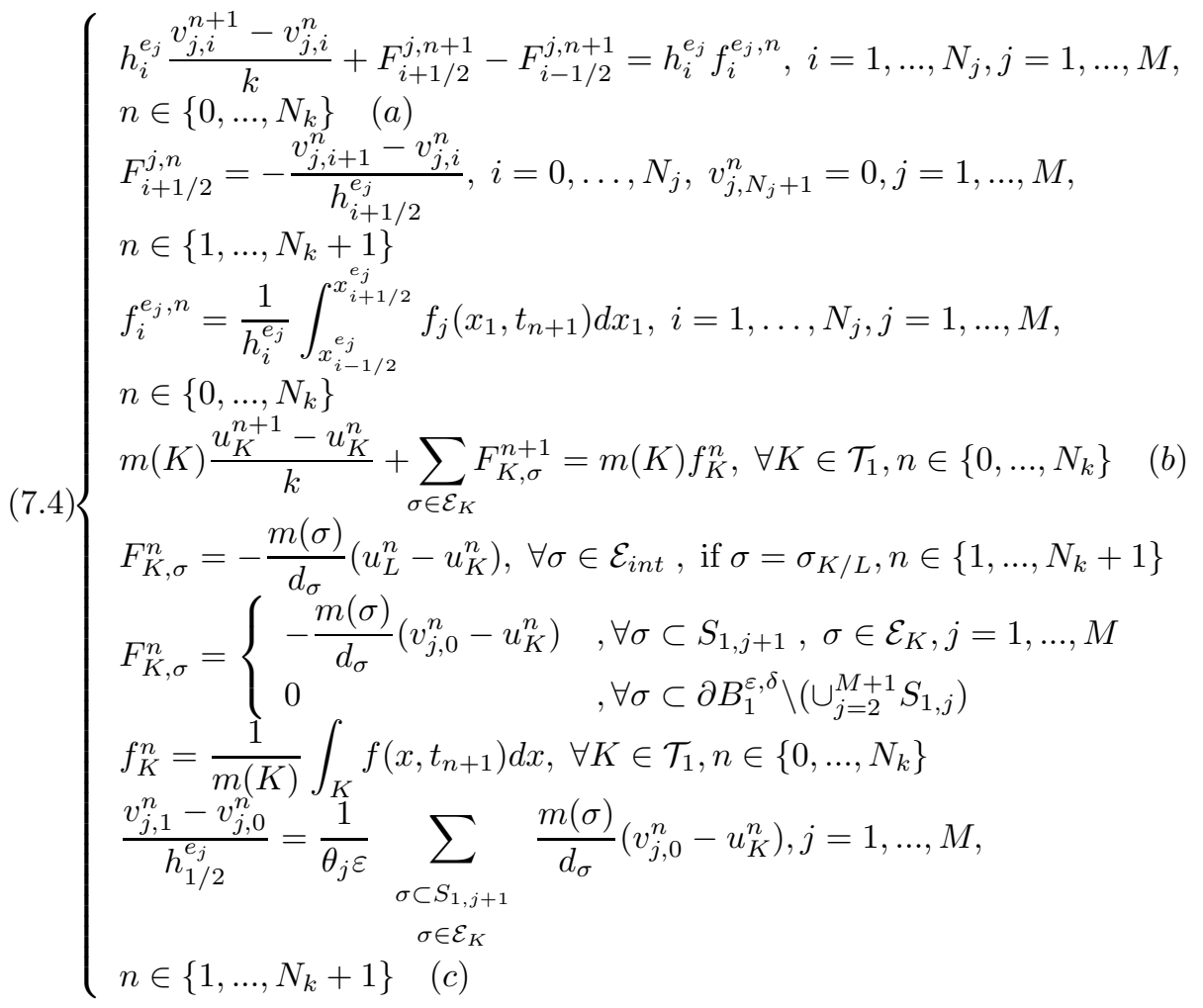

with the initial condition

$\left\{\begin{array}{l}u_{K}^{0}=0, \forall K \in \mathcal{T}_{1} \\ v_{j, i}^{0}=0, i=1, \ldots, N_{j}, j=1, \ldots, M\end{array}\right.$

Let us notice that $v_{j, 0}^{n}$ is a convex combination of the approximated values of the solution on each side of $S_{1, j+1}, j=1, \ldots, M$. This is a trivial matter to see in rewriting (7.4c) in the following way :

(7.5) $v_{j, 0}^{n}=\left(\frac{v_{j, 1}^{n}}{h_{1 / 2}^{e_{j}}}+\frac{1}{\theta_{j} \varepsilon} \sum_{\sigma \subset S_{1, j+1}, \sigma \in \mathcal{E}_{K}} \frac{m(\sigma)}{d_{\sigma}} u_{K}^{n}\right)\left(\frac{1}{h_{1 / 2}^{e_{j}}}+\frac{1}{\theta_{j} \varepsilon} \sum_{\sigma \subset S_{1, j+1}} \frac{m(\sigma)}{d_{\sigma}}\right)^{-1}$ 
For the sake of simplicity, in (7.4) and (7.5), the summation is done for $\sigma \subset S_{1, j+1}$, and for each of them, $K$ is the control volume such that $\sigma \in \mathcal{E}_{K}$.

Let us define on $B_{\varepsilon}$ the function $u_{\varepsilon, \delta, \operatorname{dec}, \mathcal{T}}^{n}$ for $n \in\left\{0, \ldots, N_{k}+1\right\}$ by

$$
u_{\varepsilon, \delta, d e c, \mathcal{T}}^{n}(x)=\left\{\begin{array}{l}
u_{K}^{n}, x \in K, K \in \mathcal{T}_{1} \\
v_{j, i}^{n}, x \in B_{1, j+1}^{d e c, \varepsilon}, x_{1}^{\left(e_{j}\right)} \in\left(x_{i-1 / 2}^{e_{j}}, x_{i+1 / 2}^{e_{j}}\right), i=1, \ldots, N_{j} \\
j=1, \ldots, M
\end{array}\right.
$$

The approximate solution of (7.2) is defined on $B_{\varepsilon} \times\left(0,\left(N_{k}+1\right) k\right)$ by $u_{\varepsilon, \delta, d e c, \mathcal{T}, k}(x, t)=u_{\varepsilon, \delta, d e c, \mathcal{T}}^{n+1}(x), x \in B_{\varepsilon}, t \in(n k,(n+1) k), n \in\left\{0, \ldots, N_{k}\right\}$

7.3. Existence and uniqueness of a solution. The scheme (7.4) leads to a linear system of the form $A U^{n+1}=B U^{n}+F^{n}$ in which $U^{n}$ is known and $U^{n+1}$ is unknown, $n \in\left\{0, \ldots, N_{k}\right\}$, the initial value $U^{0}$ being given; $\left(U^{n}\right)^{T}=\left(\left\{\left\{v_{j, i}^{n}, i=1, \ldots, N_{j}\right\}, j=1, \ldots, M\right\},\left\{u_{K}^{n}, K \in \mathcal{T}_{1}\right\}\right)$.

LEMMA 7.1. There is a unique solution $\left(U^{n+1}\right)$ to equations (7.4), $\left(U^{n}\right)$ being given.

Proof. For a given $n \in\left\{0, \ldots, N_{k}\right\}$, set $f_{i}^{e_{j}, n}=0, v_{j, i}^{n}=0, j=1, \ldots, M, i=1, \ldots, N_{j}$ and $f_{K}^{n}=0, u_{K}^{n}=0, K \in \mathcal{T}_{1}$ in (7.4). Then (7.4) becomes $A U^{n+1}=0$. Let us prove that $U^{n+1}=0$. We will deduce that $A$ is a regular matrix and that (7.4) has a unique solution.

Let us multiply (7.4a) by $v_{j, i}^{n+1}$ and sum over $i$, then multiply by $\theta_{j} \varepsilon$ and sum over $j$. In the same way, let us multiply $(7.4 \mathrm{~b})$ by $u_{K}^{n+1}$ and sum over K. We obtain

$$
\begin{aligned}
& \sum_{j=1}^{M} \theta_{j} \varepsilon \sum_{i=1}^{N_{j}} \frac{h_{i}^{e_{j}}}{k}\left(v_{j, i}^{n+1}\right)^{2}+\sum_{j=1}^{M} \theta_{j} \varepsilon \sum_{i=1}^{N_{j}}\left(F_{i+1 / 2}^{j, n+1}-F_{i-1 / 2}^{j, n+1}\right) v_{j, i}^{n+1} \\
& +\sum_{K \in \mathcal{T}_{1}} \frac{m(K)}{k}\left(u_{K}^{n+1}\right)^{2}+\sum_{K \in \mathcal{T}_{1}} \sum_{\sigma \in \mathcal{E}_{K}} F_{K, \sigma}^{n+1} u_{K}^{n+1}=0
\end{aligned}
$$

Reordering the summations over the set of edges in $B_{1}^{\varepsilon, \delta}$, considering separately the interior edges and the edges on $S_{1, j+1}, j=1, \ldots, M$, we get

$$
\begin{aligned}
& \sum_{j=1}^{M} \theta_{j} \varepsilon \sum_{i=1}^{N_{j}} \frac{h_{i}^{e_{j}}}{k}\left(v_{j, i}^{n+1}\right)^{2}+\sum_{j=1}^{M} \theta_{j} \varepsilon\left(\sum_{i=1}^{N_{j}} F_{i+1 / 2}^{j, n+1} v_{j, i}^{n+1}-\sum_{i=0}^{N_{j}-1} F_{i+1 / 2}^{j, n+1} v_{j, i+1}^{n+1}\right) \\
& +\sum_{K \in \mathcal{T}_{1}} \frac{m(K)}{k}\left(u_{K}^{n+1}\right)^{2}+\sum_{\sigma \in \mathcal{E}_{i n t}, \sigma=\sigma_{K \mid L}} F_{K, \sigma}^{n+1}\left(u_{K}^{n+1}-u_{L}^{n+1}\right) \\
& -\sum_{j=1}^{M} \sum_{\sigma \subset S_{1, j+1}, \sigma \in \mathcal{E}_{K}} \frac{m(\sigma)}{d_{\sigma}}\left(v_{j, 0}^{n+1}-u_{K}^{n+1}\right) u_{K}^{n+1}=0
\end{aligned}
$$


The subscript terms in the last summation must be read as (7.5). Replacing the numerical fluxes by their values results in

$$
\begin{aligned}
& \sum_{j=1}^{M} \theta_{j} \varepsilon \sum_{i=1}^{N_{j}} \frac{h_{i}^{e_{j}}}{k}\left(v_{j, i}^{n+1}\right)^{2}+\sum_{K \in \mathcal{T}_{1}} \frac{m(K)}{k}\left(u_{K}^{n+1}\right)^{2} \\
& +\sum_{j=1}^{M} \theta_{j} \varepsilon\left(\sum_{i=1}^{N_{j}} \frac{\left(v_{j, i+1}^{n+1}-v_{j, i}^{n+1}\right)^{2}}{h_{i+1 / 2}^{e_{j}}}+\frac{v_{j, 1}^{n+1}-v_{j, 0}^{n+1}}{h_{1 / 2}^{e_{j}}} v_{j, 1}^{n+1}\right) \\
& +\sum_{\sigma \in \mathcal{E}_{i n t}, \sigma=\sigma_{K \mid L}} \frac{m(\sigma)}{d_{\sigma}}\left(u_{K}^{n+1}-u_{L}^{n+1}\right)^{2}-\sum_{j=1}^{M} \sum_{\sigma \subset S_{1, j+1}, \sigma \in \mathcal{E}_{K}} \frac{m(\sigma)}{d_{\sigma}}\left(v_{j, 0}^{n+1}-u_{K}^{n+1}\right) u_{K}^{n+1}=0
\end{aligned}
$$

Writing (7.4c) with $n$ replaced by $n+1$, multiplying by $\theta_{j} \varepsilon v_{j, 0}^{n+1}$, summing over $j$ and adding to the above equality, lead to

$$
\begin{aligned}
& \sum_{j=1}^{M} \theta_{j} \varepsilon \sum_{i=1}^{N_{j}} \frac{h_{i}^{e_{j}}}{k}\left(v_{j, i}^{n+1}\right)^{2}+\sum_{K \in \mathcal{T}_{1}} \frac{m(K)}{k}\left(u_{K}^{n+1}\right)^{2} \\
& +\sum_{j=1}^{M} \theta_{j} \varepsilon \sum_{i=0}^{N_{j}} \frac{\left(v_{j, i+1}^{n+1}-v_{j, i}^{n+1}\right)^{2}}{h_{i+1 / 2}^{e_{j}}}+\sum_{\sigma \in \mathcal{E}_{i n t}, \sigma=\sigma_{K \mid L}} \frac{m(\sigma)}{d_{\sigma}}\left(u_{K}^{n+1}-u_{L}^{n+1}\right)^{2} \\
& +\sum_{j=1}^{M} \sum_{\sigma \subset S_{1, j+1}, \sigma \in \mathcal{E}_{K}} \frac{m(\sigma)}{d_{\sigma}}\left(v_{j, 0}^{n+1}-u_{K}^{n+1}\right)^{2}=0
\end{aligned}
$$

From (7.7), it easily follows that all the components of $U^{n+1}$ are equal. Furthermore they are equal to $v_{j, N_{j}+1}^{n+1}=0, j=1, \ldots, M$. Thereby $U^{n+1}=0$.

We must next define discrete $L^{2}$ and $H^{1}$ norms for functions on $D_{\varepsilon}$. A $H^{1}$ discrete norm was introduced in [17] in the case of a structure with a single edge. Here we propose a generalization to structures with $M$ edges.

Definition 7.2. We define $X(\mathcal{T})$ the set of the functions from $D_{\varepsilon}$ to $\mathbb{R}$ which are constant over each control volume of $\mathcal{T}$. Let $W \in X(\mathcal{T})$,

$$
W(x)=\left\{\begin{array}{l}
W_{K}, x \in K, K \in \mathcal{T}_{1} \\
W_{j, i}, x \in e_{1, j+1}^{d e c, \delta}, x_{1}^{\left(e_{j}\right)} \in\left(x_{i-1 / 2}^{e_{j}}, x_{i+1 / 2}^{e_{j}}\right), i=1, \ldots, N_{j}, j=1, \ldots, M .
\end{array}\right.
$$

We define and we denote the applications

$$
\begin{aligned}
& W \mapsto\|W\|_{2, \mathcal{T}}=\left(\sum_{K \in \mathcal{T}_{1}} m(K) W_{K}^{2}+\sum_{j=1}^{M} \theta_{j} \varepsilon \sum_{i=1}^{N_{j}} h_{i}^{e_{j}} W_{j, i}^{2}\right)^{\frac{1}{2}} \text { and } \\
& W \mapsto\|W\|_{1, \mathcal{T}}=\left(\sum_{\sigma \in \mathcal{E}_{i n t}, \sigma \subset \cup_{j=1}^{M} S_{1, j+1}} m(\sigma) d_{\sigma}\left(\frac{D_{\sigma} W}{d_{\sigma}}\right)^{2}+\sum_{j=1}^{M} \theta_{j} \varepsilon \sum_{i=0}^{N_{j}} \frac{\left(W_{j, i+1}-W_{j, i}\right)^{2}}{h_{i+1 / 2}^{e_{j}}}\right)^{1 / 2}
\end{aligned}
$$

where $D_{\sigma} W=\left\{\begin{array}{l}\left|W_{K}-W_{L}\right|, \sigma \in \mathcal{E}_{\text {int }}, \sigma=\sigma_{K \mid L} \\ \left|W_{K}-W_{j, 0}\right|, \sigma \subset S_{1, j+1}, \sigma \in \mathcal{E}_{K}, j=1, \ldots, M\end{array}\right.$

and $W_{j, 0}=\left(\frac{W_{j, 1}}{h_{1 / 2}^{e_{j}}}+\frac{1}{\theta_{j} \varepsilon} \sum_{\sigma \subset S_{1, j+1}, \sigma \in \mathcal{E}_{K}} \frac{m(\sigma)}{d_{\sigma}} W_{K}\right)\left(\frac{1}{h_{1 / 2}^{e_{j}}}+\frac{1}{\theta_{j} \varepsilon} \sum_{\sigma \subset S_{1, j+1}} \frac{m(\sigma)}{d_{\sigma}}\right)^{-1}$ 
and $W_{j, N_{j}+1}=0, j \in\{1, \ldots, M\}$.

Remark 6. For $n \in\left\{0, N_{k}+1\right\}$, let us consider the function $\underline{u}_{\varepsilon, \delta, d e c}^{n} \mathcal{T}$ defined as the restriction of $u_{\varepsilon, \delta, d e c, \mathcal{T}}^{n}$ (see (7.6)) to $D_{\varepsilon}$. Then, we remark that (7.7) can be written as $\frac{1}{k}\left\|\underline{u}_{\varepsilon, \delta, d e c, \mathcal{T}}^{n}\right\|_{2, \mathcal{T}}^{2}+\left\|\underline{u}_{\varepsilon, \delta, d e c, \mathcal{T}}^{n}\right\|_{1, \mathcal{T}}^{2}=0$.

8. Error estimate. Let $\omega_{\varepsilon, \text { poly }}^{1}$ be a polygonal domain included in $\omega_{\varepsilon}^{1}$, and $B_{\varepsilon, \text { poly }}=\left(\cup_{j=1}^{M} B_{\varepsilon}^{\left(e_{j}\right)}\right) \cup \omega_{\varepsilon, \text { poly }}^{1}$.

The boundary $\partial \omega_{\varepsilon}^{1}$ is assumed to be smooth but not necessarily plane. Consequently $\partial \omega_{\varepsilon, \text { poly }}^{1}$ and $\partial \omega_{\varepsilon}^{1}$ do not coincide, and the relation $\frac{\partial u}{\partial n}(x, t)=0$ for the solution to the partially decomposed problem is no longer true for the part of the boundary $\partial B_{1, \text { poly }}^{\varepsilon, \delta} \backslash\left(\cup_{j=1}^{M} S_{1, j+1}\right)$. Assume, that $\partial \omega_{\varepsilon, \text { poly }}^{1}$ and $\partial \omega_{\varepsilon}^{1}$ are so close that at this part of the boundary $\frac{\partial u}{\partial n}(x, t)=O(h)$.

The theorem stated below is the main result of the paper.

THEOREM 8.1. Let $u_{\varepsilon}$ be the solution of the heat equation (4.1) and $u_{\varepsilon, \delta, d e c, \mathcal{T}, k}$ be the approximate finite volume solution of the partially decomposed problem (7.2).

We denote by $h$ the size of the mesh and by $k$ the time step.

Under the assumptions of Theorem 6.2, the following estimate holds

$$
\left\|u_{\varepsilon}-u_{\varepsilon, \delta, d e c, \mathcal{T}, k}\right\|_{L^{2}\left(B_{\varepsilon, p o l y} \times(0, T)\right)}=O\left(\frac{h}{\sqrt{\varepsilon}}+k\right)+O\left(\varepsilon^{J}\right)
$$

Proof. Let $D_{\varepsilon, \text { poly }}=B_{1, \text { poly }}^{\varepsilon, \delta} \cup\left(\cup_{j=2}^{M+1} e_{1, j}^{d e c, \delta}\right)$ where $B_{1, \text { poly }}^{\varepsilon, \delta}$ is the part of $B_{\varepsilon, \text { poly }}$ truncated by the cross-sections $S_{1, j}, j=2, \ldots, M+1$, and containing $O_{1}$.

In this section, $\mathcal{T}_{1}$ is assumed to be a mesh of $B_{1, \text { poly }}^{\varepsilon, \delta}$ and $X(\mathcal{T})$ a set of functions from $D_{\varepsilon, \text { poly }}$ to $\mathbb{R}$.

Let $e_{\mathcal{T}}^{n} \in X(\mathcal{T}), n \in\left\{0, \ldots, N_{k}+1\right\}$,

$e_{\mathcal{T}}^{n}(x)=\left\{\begin{array}{l}e_{K}^{n}=u\left(x_{K}, t_{n}\right)-u_{K}^{n}, x \in K, K \in \mathcal{T}_{1} \\ e_{j, i}^{n}=v_{j}\left(x_{i}^{e_{j}}, t_{n}\right)-v_{j, i}^{n}, x \in e_{1, j}^{\text {dec, },}, x_{1}^{\left(e_{j}\right)} \in\left(x_{i-1 / 2}^{e_{j}}, x_{i+1 / 2}^{e_{j}}\right), i=1, \ldots, N_{j}, j=1, \ldots, M .\end{array}\right.$

We define $e_{j, N_{j}+1}^{n}=0$, and $e_{j, 0}^{n}$ for $j=1, \ldots, M$ by

$$
e_{j, 0}^{n}=\left(\frac{e_{j, 1}^{n}}{h_{1 / 2}^{e_{j}}}+\frac{1}{\theta_{j} \varepsilon} \sum_{\sigma \subset S_{1, j+1}, \sigma \in \mathcal{E}_{K}} \frac{m(\sigma)}{d_{\sigma}} e_{K}^{n}\right)\left(\frac{1}{h_{1 / 2}^{e_{j}}}+\frac{1}{\theta_{j} \varepsilon} \sum_{\sigma \subset S_{1, j+1}} \frac{m(\sigma)}{d_{\sigma}}\right)^{-1}
$$

Below we prove that $\left\|e_{\mathcal{T}}^{n}\right\|_{2, \mathcal{T}}=O\left(\frac{h}{\sqrt{\varepsilon}}+k\right), n \in\left\{0, \ldots, N_{k}+1\right\}$. The proof is not classical because of the interface terms relating to the consistency error on the diffusion flux when $\sigma \subset S_{1, j+1}, j=1, \ldots, M$. The choice of the initial condition implies that $\left\|e_{\mathcal{T}}^{0}\right\|_{2, \mathcal{T}}=0$. Let us remark that we will have the same result if we choose an initial condition $u_{\text {init }}$ which is other than zero and if we define $\left\{u_{K}^{0}=u_{\text {init }}\left(x_{K}\right), \forall K \in \mathcal{T}_{1}\right.$ $\left\{v_{j, i}^{0}=u_{\text {init }}\left(x_{i}^{e_{j}}\right), i=1, \ldots, N_{j}, j=1, \ldots, M\right.$

First the continuous problem (7.2) is considered. We integrate (7.2a) over each cell $I_{i}^{e_{j}}, i=1, \ldots, N_{j}, j=1, \ldots, M$, and (7.2b) over each control volume $K \in \mathcal{T}_{1}$ at time 
$t=t_{n+1}, n \in\left\{0, \ldots, N_{k}\right\}$. The choice of $f_{i}^{e_{j}, n}$ and $f_{K}^{n}$, leads to

(8.2)

$$
\left\{\begin{array}{l}
\int_{I_{i}^{e_{j}}} \frac{\partial v_{j}}{\partial t}\left(., t_{n+1}\right)+\bar{F}_{i+1 / 2}^{j, n+1}-\bar{F}_{i-1 / 2}^{j, n+1}=h_{i}^{e_{j}} f_{i}^{e_{j}, n}, i=1, \ldots, N_{j}, j=1, \ldots, M \\
n \in\left\{0, \ldots, N_{k}\right\} \\
\bar{F}_{i+1 / 2}^{j, n}=-\frac{\partial v_{j}}{\partial x_{1}^{\left(e_{j}\right)}}\left(x_{i+1 / 2}^{e_{j}}, t_{n}\right), i=0, \ldots, N_{j}, j=1, \ldots, M \\
n \in\left\{1, \ldots, N_{k}+1\right\} \\
\int_{K} \frac{\partial u}{\partial t}\left(., t_{n+1}\right)+\sum_{\sigma \in \mathcal{E}_{K}} \bar{F}_{K, \sigma}^{n+1}=m(K) f_{K}^{n}, \forall K \in \mathcal{T}_{0}, n \in\left\{0, \ldots, N_{k}\right\} \\
\bar{F}_{K, \sigma}^{n}=-\int_{\sigma} \frac{\partial u}{\partial n}\left(., t_{n}\right), \forall \sigma \in \mathcal{E}_{K}, n \in\left\{1, \ldots, N_{k}+1\right\}
\end{array}\right.
$$

At first, the consistency errors must be considered. Let us define

$$
\left\{\begin{array}{l}
F_{i+1 / 2}^{*, j, n}=-\frac{v_{j}\left(x_{i+1}^{e_{j}}, t_{n}\right)-v_{j}\left(x_{i}^{e_{j}}, t_{n}\right)}{h_{i+1 / 2}^{e_{j}}}, i=1, \ldots, N_{j}, j=1, \ldots, M \\
F_{1 / 2}^{*, j, n}=-\frac{v_{j}\left(x_{1}^{e_{j}}, t_{n}\right)-u_{j}^{*}(\delta, n)}{h_{1 / 2}^{e_{j}}}, j=1, \ldots, M
\end{array}\right.
$$

with

$$
\begin{aligned}
u_{j}^{*}(\delta, n)=\left(\frac{v_{j}\left(x_{1}^{e_{j}}, t_{n}\right)}{h_{1 / 2}^{e_{j}}}+\frac{1}{\theta_{j} \varepsilon} \sum_{\sigma \subset S_{1, j+1}, \sigma \in \mathcal{E}_{K}} \frac{m(\sigma)}{d_{\sigma}} u\left(x_{K}, t_{n}\right)\right) \\
\left(\frac{1}{h_{1 / 2}^{e_{j}}}+\frac{1}{\theta_{j} \varepsilon} \sum_{\sigma \subset S_{1, j+1}} \frac{m(\sigma)}{d_{\sigma}}\right)^{-1}
\end{aligned}
$$

In the same way, let us introduce

$(8.5) F_{K, \sigma}^{*, n}= \begin{cases}-\frac{m(\sigma)}{d_{\sigma}}\left(u\left(x_{L}, t_{n}\right)-u\left(x_{K}, t_{n}\right)\right) & , \forall \sigma \in \mathcal{E}_{\text {int }}, \text { if } \sigma=\sigma_{K / L} \\ -\frac{m(\sigma)}{d_{\sigma}}\left(u_{j}^{*}(\delta, n)-u\left(x_{K}, t_{n}\right)\right) & , \forall \sigma \subset S_{1, j+1}, \sigma \in \mathcal{E}_{K}, j=1, \ldots, M \\ 0 & , \forall \sigma \subset \partial B_{1, \text { poly }}^{\varepsilon, \delta} \backslash\left(\cup_{j=1}^{M} S_{1, j+1}\right)\end{cases}$

The consistency errors in space are called and defined by

$$
\left\{\begin{array}{l}
R_{i+1 / 2}^{j, n}=F_{i+1 / 2}^{*, j, n}-\bar{F}_{i+1 / 2}^{j, n}, i=0, \ldots, N_{j}, j=1, \ldots, M \\
R_{K, \sigma}^{n}=\frac{1}{m(\sigma)}\left(F_{K, \sigma}^{*, n}-\bar{F}_{K, \sigma}^{n}\right), \forall \sigma \in \mathcal{E}_{K}, \forall K \in \mathcal{T}_{1}
\end{array}\right.
$$

Now, let us look at the consistency errors in time for $n \in\left\{0, \ldots, N_{k}\right\}$,

$$
\left\{\begin{array}{l}
S_{i}^{j, n+1}=\int_{I_{i}^{e_{j}}} \frac{\partial v_{j}}{\partial t}\left(., t_{n+1}\right)-h_{i}^{e_{j}} \frac{v_{j}\left(x_{i}^{e_{j}}, t_{n+1}\right)-v_{j}\left(x_{i}^{e_{j}}, t_{n}\right)}{k} \\
i=1, \ldots, N_{j}, j=1, \ldots, M \\
S_{K}^{n+1}=\int_{K} \frac{\partial u}{\partial t}\left(., t_{n+1}\right)-m(K) \frac{u\left(x_{K}, t_{n+1}\right)-u\left(x_{K}, t_{n}\right)}{k}, \forall K \in \mathcal{T}_{1}
\end{array}\right.
$$


Although $R_{K, \sigma}^{n} \neq 0$ if $\sigma \in \mathcal{E}_{K}, \sigma \subset \partial B_{1, \text { poly }}^{\varepsilon, \delta} \backslash\left(\cup_{j=1}^{M} S_{1, j+1}\right), R_{K, \sigma}^{n}$ is at least of the order of $h$ on $\partial B_{1, \text { poly }}^{\varepsilon, \delta} \backslash\left(\cup_{j=1}^{M} S_{1, j+1}\right)$. And so, the following estimates hold.

$$
\left\{\begin{array}{l}
R_{i+1 / 2}^{j, n}=O\left(h\left\|\frac{\partial^{2} v_{j}}{\partial x_{1}^{\left(e_{j}\right) 2}}\right\|_{\infty}\right), i=1, \ldots, N_{j}, j=1, \ldots, M \\
R_{K, \sigma}^{n}=O\left(h\left\|\nabla^{2} u\right\|_{\infty}\right), \forall \sigma \in \mathcal{E}_{K} \cap \mathcal{E}_{i n t}, \forall K \in \mathcal{T}_{1} \\
R_{K, \sigma}^{n}=O\left(h\left\|\nabla^{2} u\right\|_{\infty}\right), \forall \sigma \in \mathcal{E}_{K}, \forall \sigma \subset \partial B_{1, \text { poly }}^{\varepsilon, \delta} \backslash\left(\cup_{j=1}^{M} S_{1, j+1}\right), \forall K \in \mathcal{T}_{1} \\
S_{i}^{j, n+1}=h_{i}^{e_{j}} O\left(h\left\|\frac{\partial^{2} v_{j}}{\partial t \partial x_{1}^{\left(e_{j}\right)}}\right\|_{\infty}+k\left\|\frac{\partial^{2} v_{j}}{\partial t^{2}}\right\|_{\infty}\right), i=1, \ldots, N_{j}, \\
j=1, \ldots, M \\
\left.S_{K}^{n+1}=m(K) O\left(k\left\|\frac{\partial^{2} u}{\partial t^{2}}\right\|_{\infty}\right)+h\left\|\frac{\partial}{\partial t} \nabla u\right\|_{\infty}\right), \forall K \in \mathcal{T}_{0}
\end{array}\right.
$$

Now, in order to evaluate the consistency errors on the interfaces, let us define the following quantities for $j=1, \ldots, M$, and for all $\sigma \subset S_{1, j+1}$ such that $\sigma \in \mathcal{E}_{K}$ for some $K \in \mathcal{T}_{1}$

$$
\left\{\begin{array}{l}
R_{1 / 2}^{*, j, n}=-\frac{v_{j}\left(\delta, t_{n}\right)-u_{j}^{*}(\delta, n)}{h_{1 / 2}^{e_{j}}} \\
R_{1 / 2}^{\nabla, j, n}=-\frac{v_{j}\left(x_{1}^{e_{j}}, t_{n}\right)-v_{j}\left(\delta, t_{n}\right)}{h_{1 / 2}^{e_{j}}}+\frac{\partial v_{j}}{\partial x_{1}^{\left(e_{j}\right)}}\left(\delta, t_{n}\right) \\
R_{K, \sigma}^{*, n}=-\frac{u_{j}^{*}(\delta, n)-v_{j}\left(\delta, t_{n}\right)}{d_{\sigma}} \\
R_{K, \sigma}^{\nabla, n}=-\frac{v_{j}\left(\delta, t_{n}\right)-u\left(x_{K}, t_{n}\right)}{d_{\sigma}}+\frac{1}{m(\sigma)} \int_{\sigma} \frac{\partial u}{\partial n}\left(., t_{n}\right) d \gamma
\end{array}\right.
$$

such that, for $j=1, \ldots, M$, and for all $\sigma \in \mathcal{E}_{K}, \sigma \subset S_{1, j+1}$,

$$
R_{1 / 2}^{j, n}=R_{1 / 2}^{*, j, n}+R_{1 / 2}^{\nabla, j, n}, \quad R_{K, \sigma}^{n}=R_{K, \sigma}^{*, n}+R_{K, \sigma}^{\nabla, n}
$$

The estimates

$$
R_{1 / 2}^{\nabla, j, n}=O\left(h\left\|\frac{\partial^{2} v_{j}}{\partial x_{1}^{\left(e_{j}\right) 2}}\right\|_{\infty}\right), \quad R_{K, \sigma}^{\nabla, n}=O\left(h\left\|\nabla^{2} u\right\|_{\infty}\right)
$$

hold for $j=1, \ldots, M$, and for all $\sigma \in \mathcal{E}_{K}, \sigma \subset S_{1, j+1}$, whereas any estimate is comparable for $R_{1 / 2}^{*, j, n}$ and $R_{K, \sigma}^{*, n}$. The result below stems from (8.4) and (7.2c).

$$
\sum_{\sigma \subset S_{1, j+1}, \sigma \in \mathcal{E}_{K}} m(\sigma) R_{K, \sigma}^{n}-\theta_{j} \varepsilon R_{1 / 2}^{j, n}=0, j=1, \ldots, M
$$

It was stated in [17] in the case $M=1$. It holds the key to simplifying the terms $R_{1 / 2}^{*, j, n}$ and $R_{K, \sigma}^{*, n}$ in (8.19).

Thereafter, the proof is continued by subtracting the corresponding equations of (7.4) and (8.2). This results in 


$$
\left\{\begin{array}{l}
\int_{I_{i}^{e_{j}}} \frac{\partial v_{j}}{\partial t}\left(., t_{n+1}\right)-h_{i}^{e_{j}} \frac{v_{j, i}^{n+1}-v_{j, i}^{n}}{k}+\bar{F}_{i+1 / 2}^{j, n+1}-F_{i+1 / 2}^{j, n+1} \\
-\left(\bar{F}_{i-1 / 2}^{j, n+1}-F_{i-1 / 2}^{j, n+1}\right)=0, i=1, \ldots, N_{j}, j=1, \ldots, M, n \in\left\{0, \ldots, N_{k}\right\} \\
\int_{K} \frac{\partial u}{\partial t}\left(., t_{n+1}\right)-m(K) \frac{u_{K}^{n+1}-u_{K}^{n}}{k}+\sum_{\sigma \in \mathcal{E}_{K}}\left(\bar{F}_{K, \sigma}^{n+1}-F_{K, \sigma}^{n+1}\right)=0 \\
\forall K \in \mathcal{T}_{1}, n \in\left\{0, \ldots, N_{k}\right\}
\end{array}\right.
$$

The consistency errors are then highlighted. We get with (8.6) and (8.7)

$$
\left\{\begin{array}{l}
S_{i}^{j, n+1}+h_{i}^{e_{j}} \frac{e_{j, i}^{n+1}-e_{j, i}^{n}}{k}+F_{i+1 / 2}^{*, j, n+1}-F_{i+1 / 2}^{j, n+1}-\left(F_{i-1 / 2}^{*, j, n+1}-F_{i-1 / 2}^{j, n+1}\right) \\
-\left(R_{i+1 / 2}^{j, n+1}-R_{i-1 / 2}^{j, n+1}\right)=0, i=1, \ldots, N_{j}, j=1, \ldots, M \\
S_{K}^{n+1}+m(K) \frac{e_{K}^{n+1}-e_{K}^{n}}{k}+\sum_{\sigma \in \mathcal{E}_{K}}\left(F_{K, \sigma}^{*, n+1}-F_{K, \sigma}^{n+1}\right) \\
-\sum_{\sigma \in \mathcal{E}_{K}} m(\sigma) R_{K, \sigma}^{n+1}=0, \forall K \in \mathcal{T}_{1}, n \in\left\{0, \ldots, N_{k}\right\}
\end{array}\right.
$$

Let us remark that $e_{j, 0}^{n+1}=u_{j}^{*}(\delta, n+1)-v_{j, 0}^{n+1}$ and that

$$
\left\{\begin{array}{l}
F_{i+1 / 2}^{*, j, n+1}-F_{i+1 / 2}^{j, n+1}=-\frac{e_{j, i+1}^{n+1}-e_{j, i}^{n+1}}{h_{i+1 / 2}^{e_{j}}}, i=0, \ldots, N_{j}, j=1, \ldots, M \\
F_{K, \sigma}^{*, n+1}-F_{K, \sigma}^{n+1}= \begin{cases}-\frac{m(\sigma)}{d_{\sigma}}\left(e_{L}^{n+1}-e_{K}^{n+1}\right) & , \forall \sigma \in \mathcal{E}_{i n t}, \text { if } \sigma=\sigma_{K / L} \\
-\frac{m(\sigma)}{d_{\sigma}}\left(e_{j, 0}^{n+1}-e_{K}^{n+1}\right) & , \forall \sigma \subset S_{1, j+1}, \sigma \in \mathcal{E}_{K}, j=1, \ldots, M \\
0 & , \forall \sigma \subset \partial B_{1, \text { poly }}^{\varepsilon, \delta} \backslash\left(\cup_{j=1}^{M} S_{1, j+1}\right)\end{cases}
\end{array}\right.
$$

Let us denote by $\delta_{\Gamma}$ the function such that $\delta_{\Gamma}(\sigma)=1$ if $\sigma \subset \Gamma$ (if $\Gamma$ is a part of the boundary), or if $\sigma \in \Gamma$ (if $\Gamma$ is a set of edges), and 0 elsewhere. Next, the above expressions are replaced in (8.14).

$$
\left\{\begin{array}{l}
h_{i}^{e_{j}} \frac{e_{j, i}^{n+1}-e_{j, i}^{n}}{k}-\frac{e_{j, i+1}^{n+1}-e_{j, i}^{n+1}}{h_{i+1 / 2}^{e_{j}}}+\frac{e_{j, i}^{n+1}-e_{j, i-1}^{n+1}}{h_{i-1 / 2}^{e_{j}}} \\
=R_{i+1 / 2}^{j, n+1}-R_{i-1 / 2}^{j, n+1}-S_{i}^{j, n+1}, i=1, \ldots, N_{j}, j=1, \ldots, M \\
m(K) \frac{e_{K}^{n+1}-e_{K}^{n}}{k}-\sum_{\sigma \in \mathcal{E}_{K}} \sum_{j=1}^{M} \delta_{S_{1, j+1}}(\sigma) \frac{m(\sigma)}{d_{\sigma}}\left(e_{j, 0}^{n+1}-e_{K}^{n+1}\right) \\
-\sum_{\sigma \in \mathcal{E}_{K}} \delta_{\mathcal{E}_{i n t}}(\sigma) \frac{m(\sigma)}{d_{\sigma}}\left(e_{L}^{n+1}-e_{K}^{n+1}\right) \\
=\sum_{\sigma \in \mathcal{E}_{K}} m(\sigma) R_{K, \sigma}^{n+1}-S_{K}^{n+1}, \forall K \in \mathcal{T}_{1}
\end{array}\right.
$$

Multiplying (8.15a) by $e_{j, i}^{n+1}$, summing over $i$, multiplying again by $\theta_{j} \varepsilon$, and summing 
over $j$, yield

$$
\begin{aligned}
& \sum_{j=1}^{M} \theta_{j} \varepsilon \sum_{i=1}^{N_{j}} h_{i}^{e_{j}} \frac{\left(e_{j, i}^{n+1}\right)^{2}}{k}+\sum_{j=1}^{M} \theta_{j} \varepsilon \sum_{i=1}^{N_{j}} \frac{\left(e_{j, i+1}^{n+1}-e_{j, i}^{n+1}\right)^{2}}{h_{i+1 / 2}^{e_{j}}} \\
& +\sum_{j=1}^{M} \theta_{j} \varepsilon \frac{e_{j, 1}^{n+1}-e_{j, 0}^{n+1}}{h_{1 / 2}^{e_{j}}} e_{j, 1}^{n+1} \\
& =\sum_{j=1}^{M} \theta_{j} \varepsilon \sum_{i=1}^{N_{j}} h_{i}^{e_{j}} \frac{e_{j, i}^{n} e_{j, i}^{n+1}}{k}+\sum_{j=1}^{M} \theta_{j} \varepsilon \sum_{i=1}^{N_{j}} R_{i+1 / 2}^{j, n+1}\left(e_{j, i}^{n+1}-e_{j, i+1}^{n+1}\right) \\
& -\sum_{j=1}^{M} \theta_{j} \varepsilon R_{1 / 2}^{j, n+1} e_{j, 1}^{n+1}-\sum_{j=1}^{M} \theta_{j} \varepsilon \sum_{i=1}^{N_{j}} S_{i}^{j, n+1} e_{j, i}^{n+1}
\end{aligned}
$$

Multiplying (8.15b) by $e_{K}^{n+1}$, summing over $K \in \mathcal{T}_{1}$, reordering the summations, and summing over $j$, lead to

$$
\begin{aligned}
& \sum_{K \in \mathcal{T}_{1}} m(K) \frac{\left(e_{K}^{n+1}\right)^{2}}{k}+\sum_{\sigma \in \mathcal{E}_{i n t}, \sigma=\sigma_{K / L}} \frac{m(\sigma)}{d_{\sigma}}\left(e_{L}^{n+1}-e_{K}^{n+1}\right)^{2} \\
& -\sum_{j=1}^{M} \sum_{\sigma \subset S_{1, j+1}, \sigma \in \mathcal{E}_{K}} \frac{m(\sigma)}{d_{\sigma}}\left(e_{j, 0}^{n+1}-e_{K}^{n+1}\right) e_{K}^{n+1}=\sum_{K \in \mathcal{T}_{1}} m(K) \frac{e_{K}^{n} e_{K}^{n+1}}{k} \\
& +\sum_{\sigma \in \mathcal{E}_{i n t}, \sigma=\sigma_{K / L}} m(\sigma) R_{K, \sigma}^{n+1}\left(e_{K}^{n+1}-e_{L}^{n+1}\right)+\sum_{j=1}^{M} \sum_{\sigma \subset S_{1, j+1}, \sigma \in \mathcal{E}_{K}} m(\sigma) R_{K, \sigma}^{n+1} e_{K}^{n+1} \\
& -\sum_{K \in \mathcal{T}_{1}} S_{K}^{n+1} e_{K}^{n+1}
\end{aligned}
$$

On the boundary, the summations are done for $\sigma \subset S_{1, j+1}$, and for each of them, $K$ is the control volume such that $\sigma \in \mathcal{E}_{K}$ (as in (7.5)).

Thereafter, we add (8.16) and (8.17). First, the result of adding the expressions, on the left hand side with terms on the interfaces, is detailed. From (8.1), it follows that

$$
\begin{aligned}
& \theta_{j} \varepsilon \frac{e_{j, 1}^{n+1}-e_{j, 0}^{n+1}}{h_{1 / 2}^{e_{j}}} e_{j, 1}^{n+1}-\sum_{\sigma \subset S_{1, j+1}, \sigma \in \mathcal{E}_{K}} \frac{m(\sigma)}{d_{\sigma}}\left(e_{j, 0}^{n+1}-e_{K}^{n+1}\right) e_{K}^{n+1} \\
& =\theta_{j} \varepsilon \frac{\left(e_{j, 1}^{n+1}-e_{j, 0}^{n+1}\right)^{2}}{h_{1 / 2}^{e_{j}}}+\sum_{\sigma \subset S_{1, j+1}, \sigma \in \mathcal{E}_{K}} \frac{m(\sigma)}{d_{\sigma}}\left(e_{j, 0}^{n+1}-e_{K}^{n+1}\right)^{2}
\end{aligned}
$$

Second, the expressions, on the right hand side of (8.16) and (8.17) with terms on the interfaces, are added. From (8.12), this results in

$$
\begin{aligned}
& -\theta_{j} \varepsilon R_{1 / 2}^{j, n+1} e_{j, 1}^{n+1}+\sum_{\sigma \subset S_{1, j+1}, \sigma \in \mathcal{E}_{K}} m(\sigma) R_{K, \sigma}^{n+1} e_{K}^{n+1} \\
(8.19)= & -\theta_{j} \varepsilon R_{1 / 2}^{j, n+1}\left(e_{j, 1}^{n+1}-e_{j, 0}^{n+1}\right)+\sum_{\sigma \subset S_{1, j+1, \sigma \in \mathcal{E}_{K}}} m(\sigma) R_{K, \sigma}^{n+1}\left(e_{K}^{n+1}-e_{j, 0}^{n+1}\right) \\
= & -\theta_{j} \varepsilon R_{1 / 2}^{\nabla, j, n+1}\left(e_{j, 1}^{n+1}-e_{j, 0}^{n+1}\right)+\sum_{\sigma \subset S_{1, j+1}, \sigma \in \mathcal{E}_{K}} m(\sigma) R_{K, \sigma}^{\nabla, n+1}\left(e_{K}^{n+1}-e_{j, 0}^{n+1}\right)
\end{aligned}
$$

Indeed, it can be seen through (8.1) that the expression above is equal to zero if we replace $R^{\nabla}$ by $R^{*}$. 
Now, summing (8.16) and (8.17) yields

$$
\begin{aligned}
& \frac{1}{k}\left\|e_{\mathcal{T}}^{n+1}\right\|_{2, \mathcal{T}}^{2}+\left\|e_{\mathcal{T}}^{n+1}\right\|_{1, \mathcal{T}}^{2}=\sum_{j=1}^{M} \theta_{j} \varepsilon \sum_{i=1}^{N_{j}} h_{i}^{e_{j}} \frac{e_{j, i}^{n} e_{j, i}^{n+1}}{k}+\sum_{K \in \mathcal{T}_{1}} m(K) \frac{e_{K}^{n} e_{K}^{n+1}}{k} \\
& +\sum_{j=1}^{M} \theta_{j} \varepsilon \sum_{i=1}^{N_{j}} R_{i+1 / 2}^{j, n+1}\left(e_{j, i}^{n+1}-e_{j, i+1}^{n+1}\right)-\sum_{j=1}^{M} \theta_{j} \varepsilon R_{1 / 2}^{\nabla, j, n+1}\left(e_{j, 1}^{n+1}-e_{j, 0}^{n+1}\right) \\
& +\sum_{j=1}^{M} \sum_{\sigma \subset S_{1, j+1}, \sigma \in \mathcal{E}_{K}} m(\sigma) R_{K, \sigma}^{\nabla, n+1}\left(e_{K}^{n+1}-e_{j, 0}^{n+1}\right)-\sum_{K \in \mathcal{T}_{1}} S_{K}^{n+1} e_{K}^{n+1} \\
& +\sum_{\sigma \in \mathcal{E}_{i n t}, \sigma=\sigma_{K / L}} m(\sigma) R_{K, \sigma}^{n+1}\left(e_{K}^{n+1}-e_{L}^{n+1}\right)-\sum_{j=1}^{M} \theta_{j} \varepsilon \sum_{i=1}^{N_{j}} S_{i}^{j, n+1} e_{j, i}^{n+1}
\end{aligned}
$$

Next, we will distinguish three categories among the right terms and estimate each of them : the terms relating to the consistency errors in space, those relating to the consistency errors in time, and the remaining terms.

To begin with, an estimate of the terms relating to the consistency errors in space is provided. On the basis of the estimates (8.8) and (8.11) and the Cauchy-Schwarz inequality, there exists a constant $C_{1}$ such that

$$
\begin{aligned}
& \mid \sum_{j=1}^{M} \theta_{j} \varepsilon \sum_{i=1}^{N_{j}} R_{i+1 / 2}^{j, n+1}\left(e_{j, i}^{n+1}-e_{j, i+1}^{n+1}\right)-\sum_{j=1}^{M} \theta_{j} \varepsilon R_{1 / 2}^{\nabla, j, n+1}\left(e_{j, 1}^{n+1}-e_{j, 0}^{n+1}\right) \\
& +\sum_{j=1}^{M} \sum_{\sigma \subset S_{1, j+1}, \sigma \in \mathcal{E}_{K}} m(\sigma) R_{K, \sigma}^{\nabla, n+1}\left(e_{K}^{n+1}-e_{j, 0}^{n+1}\right) \\
& +\sum_{\sigma \in \mathcal{E}_{i n t}, \sigma=\sigma_{K / L}} m(\sigma) R_{K, \sigma}^{n+1}\left(e_{K}^{n+1}-e_{L}^{n+1}\right) \mid \\
& \leq\left(\sum_{j=1}^{M} \theta_{j} \varepsilon\left(\sum_{i=1}^{N_{j}}\left(R_{i+1 / 2}^{j, n+1}\right)^{2} h_{i+1 / 2}^{e_{j}}+\left(R_{1 / 2}^{\nabla, j, n+1}\right)^{2} h_{1 / 2}^{e_{j}}\right)\right. \\
& \left.+\sum_{\sigma \in \mathcal{E}_{i n t}, \sigma=\sigma_{K / L}} m(\sigma) d_{\sigma}\left(R_{K, \sigma}^{n+1}\right)^{2}+\sum_{j=1}^{M} \sum_{\sigma \subset S_{1, j+1}, \sigma \in \mathcal{E}_{K}} m(\sigma) d_{\sigma}\left(R_{K, \sigma}^{\nabla, n+1}\right)^{2}\right)^{1 / 2}\left\|e_{\mathcal{T}}^{n+1}\right\|_{1, \mathcal{T}} \\
& \leq C_{1} h\left(\max _{j=1, \ldots, M}\left\|\frac{\partial^{2} v_{j}}{\partial x_{1}^{\left(e_{j}\right) 2}}\right\|_{\infty}+\left\|\nabla^{2} u\right\|_{\infty}\right)\left(\sum_{j=1}^{M} \theta_{j} \varepsilon\left(l_{j}-\delta\right)+2 \sum_{j=0}^{p} m\left(B_{1, \text { poly }}^{\varepsilon, \delta}\right)^{1 / 2}\left\|e_{\mathcal{T}}^{n+1}\right\|_{1, \mathcal{T}}\right.
\end{aligned}
$$

We proved in Theorem 6.3 that $\left\|\frac{\partial^{2} v_{j}}{\partial x_{1}^{\left(e_{j}\right) 2}}\right\|_{\infty}=O(1), j=1, \ldots, M$, and $\left\|\nabla^{2} u\right\|_{\infty}=$ $O\left(\frac{1}{\varepsilon}\right)$. Let us remind that $m\left(B_{1, \text { poly }}^{\varepsilon, \delta}\right)=O(\varepsilon \delta)$. Hence, there exists a constant $C_{2}$ such that the terms relating to the consistency errors in space in (8.20) are bounded by

$$
\begin{aligned}
& \leq C_{2} \frac{h}{\sqrt{\varepsilon}}\left\|e_{\mathcal{T}}^{n+1}\right\|_{1, \mathcal{T}} \\
& \leq\left\|e_{\mathcal{T}}^{n+1}\right\|_{1, \mathcal{T}}^{2}+O\left(\frac{h^{2}}{\varepsilon}\right)
\end{aligned}
$$


In turn, the second category of terms in (8.20) is studied. From (8.8), the terms in (8.20) relating to the consistency errors in time are bounded in the following way

$$
\begin{aligned}
& \left|-\sum_{K \in \mathcal{T}_{1}} S_{K}^{n+1} e_{K}^{n+1}-\sum_{j=1}^{M} \theta_{j} \varepsilon \sum_{i=1}^{N_{j}} S_{i}^{j, n+1} e_{j, i}^{n+1}\right| \\
& \leq O\left(k\left\|\frac{\partial^{2} u}{\partial t^{2}}\right\|_{\infty}+h\left\|\frac{\partial}{\partial t} \nabla u\right\|_{\infty}\right) \sum_{K \in \mathcal{T}_{1}} m(K)\left|e_{K}^{n+1}\right|+O(h+k) \sum_{j=1}^{M} \theta_{j} \varepsilon \sum_{i=1}^{N_{j}} h_{i}^{e_{j}}\left|e_{j, i}^{n+1}\right|
\end{aligned}
$$

The estimates $\left\|\frac{\partial^{2} u}{\partial t^{2}}\right\|_{\infty}=O(1), j=0, \ldots, M$, and $\left\|\frac{\partial}{\partial t} \nabla u\right\|_{\infty}=O(1)$ result from Section 6 . Then the Cauchy-Schwarz inequality is used to prove that the terms relating to the consistency errors in time in (8.20) are bounded by

$$
\leq O(\sqrt{\varepsilon}) O(h+k)\left\|e_{\mathcal{T}}^{n+1}\right\|_{2, \mathcal{T}}
$$

Thereafter, we look at the third category of terms in (8.20) which are the two first terms on the right hand side. We have

$$
\begin{aligned}
& \left|\sum_{j=1}^{M} \theta_{j} \varepsilon \sum_{i=1}^{N_{j}} h_{i}^{e_{j}} \frac{e_{j, i}^{n} e_{j, i}^{n+1}}{k}+\sum_{K \in \mathcal{T}_{1}} m(K) \frac{e_{K}^{n} e_{K}^{n+1}}{k}\right| \\
& \leq \frac{1}{2 k}\left\|e_{\mathcal{T}}^{n+1}\right\|_{2, \mathcal{T}}^{2}+\frac{1}{2 k}\left\|e_{\mathcal{T}}^{n}\right\|_{2, \mathcal{T}}^{2}
\end{aligned}
$$

that yields eventually, with (8.21) and (8.22), the following bound for (8.20)

$$
\begin{aligned}
& \frac{1}{k}\left\|e_{\mathcal{T}}^{n+1}\right\|_{2, \mathcal{T}}^{2}+\left\|e_{\mathcal{T}}^{n+1}\right\|_{1, \mathcal{T}}^{2} \leq \frac{1}{2 k}\left\|e_{\mathcal{T}}^{n+1}\right\|_{2, \mathcal{T}}^{2}+\frac{1}{2 k}\left\|e_{\mathcal{T}}^{n}\right\|_{2, \mathcal{T}}^{2} \\
& +O(\sqrt{\varepsilon}) O(h+k)\left\|e_{\mathcal{T}}^{n+1}\right\|_{2, \mathcal{T}}+\left\|e_{\mathcal{T}}^{n+1}\right\|_{1, \mathcal{T}}^{2}+O\left(\frac{h^{2}}{\varepsilon}\right)
\end{aligned}
$$

Consequently

$$
\left\|e_{\mathcal{T}}^{n+1}\right\|_{2, \mathcal{T}}^{2} \leq\left\|e_{\mathcal{T}}^{n}\right\|_{2, \mathcal{T}}^{2}+2 k O(\sqrt{\varepsilon}) O(h+k)\left\|e_{\mathcal{T}}^{n+1}\right\|_{2, \mathcal{T}}+k O\left(\frac{h^{2}}{\varepsilon}\right)
$$

Next, the Young inequality, that is to say : $2 a b \leq(\xi a)^{2}+\left(\frac{b}{\xi}\right)^{2}$ with $\xi^{2}=\frac{k+1}{k}$, for real $a, b$, enables us to provethat there exists a constant $C_{3}$ such that

$$
\begin{aligned}
& \left\|e_{\mathcal{T}}^{n+1}\right\|_{2, \mathcal{T}}^{2} \leq(k+1)\left\|e_{\mathcal{T}}^{n}\right\|_{2, \mathcal{T}}^{2}+k(k+1)^{2} O(\varepsilon)(O(h+k))^{2}+k O\left(\frac{h^{2}}{\varepsilon}\right) \\
& \leq(k+1)\left\|e_{\mathcal{T}}^{n}\right\|_{2, \mathcal{T}}^{2}+C_{3} k\left(\frac{h}{\sqrt{\varepsilon}}+k\right)^{2}
\end{aligned}
$$

The following step is devoted to demonstrating by induction on $n$ an estimate of $\left\|e_{\mathcal{T}}^{n}\right\|_{2, \mathcal{T}}^{2}$. At first, the sequence $\left(\alpha_{n}\right)_{n \geq 0}$ by $\alpha_{0}=0, \alpha_{n+1}=(k+1) \alpha_{n}+C_{3} k, n \geq 0$, is considered. It is easy to see that $\left\|e_{\mathcal{T}}^{n}\right\|_{2, \mathcal{T}}^{2} \leq \alpha_{n}\left(\frac{h}{\sqrt{\varepsilon}}+k\right)^{2}$ leads to $\left\|e_{\mathcal{T}}^{n+1}\right\|_{2, \mathcal{T}}^{2} \leq$ $\alpha_{n+1}\left(\frac{h}{\sqrt{\varepsilon}}+k\right)^{2}$. Since $\left\|e_{\mathcal{T}}^{0}\right\|_{2, \mathcal{T}}^{2}=0$, the following estimate holds

$$
\forall n \in\left\{0, \ldots, N_{k}+1\right\},\left\|e_{\mathcal{T}}^{n}\right\|_{2, \mathcal{T}}^{2} \leq \alpha_{n}\left(\frac{h}{\sqrt{\varepsilon}}+k\right)^{2}
$$


Let us bound $\alpha_{n}$ :

$\alpha_{n}=C_{3} k\left((1+k)^{n}-1\right) \leq C_{3} k\left(\left(e^{k}\right)^{n}-1\right) \leq C_{3} k e^{2 n k} \leq C_{3} k e^{4 T}$

This yields

$$
\forall n \in\left\{0, \ldots, N_{k}+1\right\},\left\|e_{\mathcal{T}}^{n}\right\|_{2, \mathcal{T}}=O\left(\frac{h}{\sqrt{\varepsilon}}+k\right)
$$

which is the central findings of the proof. Now, according to Theorem 6.2, the estimate of Theorem 8.1 follows as soon as we can show that

$$
\left\|u_{\varepsilon, \delta, d e c}-u_{\varepsilon, \delta, \operatorname{dec}, \mathcal{T}, k}\right\|_{L^{2}\left(B_{\varepsilon, \text { poly }} \times(0, T)\right)}=O\left(\frac{h}{\sqrt{\varepsilon}}+k\right)
$$

The remaining part of the proof is devoted to prove (8.26). To this end, the norm is divided into two parts

$$
\begin{aligned}
& \left\|u_{\varepsilon, \delta, \operatorname{dec}}-u_{\varepsilon, \delta, \operatorname{dec}, \mathcal{T}, k}\right\|_{L^{2}\left(B_{\varepsilon, \mathrm{poly}} \times(0, T)\right)} \\
& \leq\left\|u_{\varepsilon, \delta, \operatorname{dec}}-\tilde{u}_{\varepsilon, \delta, \operatorname{dec}, \mathcal{T}, k}\right\|_{L^{2}\left(B_{\varepsilon, \text { poly }} \times(0, T)\right)}+\left\|\tilde{u}_{\varepsilon, \delta, \operatorname{dec}, \mathcal{T}, k}-u_{\varepsilon, \delta, \operatorname{dec}, \mathcal{T}, k}\right\|_{L^{2}\left(B_{\varepsilon, \text { poly }} \times(0, T)\right)}
\end{aligned}
$$

Before defining $\tilde{u}_{\varepsilon, \delta, \operatorname{dec}, \mathcal{T}, k}$, let us start introducing the function $\tilde{u}_{\varepsilon, \delta, \text { dec, } \mathcal{T}}^{n}$ for $n \in$ $\left\{0, \ldots, N_{k}+1\right\}$ defined on $B_{\varepsilon, \text { poly }}$ by

$$
\tilde{u}_{\varepsilon, \delta, \operatorname{dec}, \mathcal{T}}^{n}(x)=\left\{\begin{array}{l}
u\left(x_{K}, t^{n}\right), x \in K, K \in \mathcal{T}_{1} \\
v_{j}\left(x_{i}^{e_{j}}, t^{n}\right), x \in B_{1, j+1}^{d e c, \varepsilon}, x_{1}^{\left(e_{j}\right)} \in\left(x_{i-1 / 2}^{e_{j}}, x_{i+1 / 2}^{e_{j}}\right), \\
i=1, \ldots, N_{j}, j=1, \ldots, M .
\end{array}\right.
$$

Next, let $\underline{\tilde{u}}_{\varepsilon, \delta, d e c, \mathcal{T}}^{n}$ be the restriction of $\tilde{u}_{\varepsilon, \delta, d e c, \mathcal{T}}^{n}$ to $D_{\varepsilon, \text { poly }}$. By so doing, we have

$$
e_{\mathcal{T}}^{n}=\underline{\tilde{u}}_{\varepsilon, \delta, \operatorname{dec}, \mathcal{T}}^{n}-\underline{u}_{\varepsilon, \delta, \operatorname{dec}, \mathcal{T}}^{n}
$$

Eventually, let us define

$$
\tilde{u}_{\varepsilon, \delta, d e c, \mathcal{T}, k}(x, t)=\tilde{u}_{\varepsilon, \delta, d e c, \mathcal{T}}^{n+1}(x), x \in B_{\varepsilon, \text { poly }}, t \in(n k,(n+1) k), n \in\left\{0, \ldots, N_{k}\right\}
$$

For (8.26) to stand, we must next estimate the two parts above. For one

$$
\begin{aligned}
& \left\|\tilde{u}_{\varepsilon, \delta, \operatorname{dec}, \mathcal{T}, k}-u_{\varepsilon, \delta, \operatorname{dec}, \mathcal{T}, k}\right\|_{L^{2}\left(B_{\varepsilon, \text { poly }} \times(0, T)\right)}^{2} \\
& \leq \sum_{n=0}^{N_{k}} k\left\|e_{\mathcal{T}}^{n+1}\right\|_{2, \mathcal{T}}^{2} \\
& \leq 2 T \max \left\{\left\|e_{\mathcal{T}}^{n+1}\right\|_{2, \mathcal{T}}^{2}, 0 \leq n \leq N_{k}\right\} \\
& =O\left(\frac{h}{\sqrt{\varepsilon}}+k\right)^{2}
\end{aligned}
$$

For another, using Theorem 6.3, we know that the first partial derivative both in space and time of $u$ and $v_{j}, j=1, \ldots, M$, are bounded, and we deduce that

$$
\left\|u_{\varepsilon, \delta, d e c}-\tilde{u}_{\varepsilon, \delta, d e c, \mathcal{T}, k}\right\|_{L^{2}\left(B_{\varepsilon, \text { poly }} \times(0, T)\right)}=O((h+k) \sqrt{\varepsilon})
$$

so this term is negligible with respect to the previous one. So (8.26) is proved. 
REMARK 7. A comparison between the hybrid scheme used on a dimensionallyheterogeneous domain and the so-called TPFA scheme (defined in [18]) used on a full bi-dimensional non-matching finite volume mesh, solving the Poisson equation in a rod structure with a single node and a single branch, may be found in [17]. The branch is of thickness $\varepsilon$, and meshed with a row of rectangular cells $\varepsilon$ high by $h$ wide, where $h$ is the size of the mesh of the remaining part of the domain (corresponding to the node). The a priori estimate on the error which is achieved in [17] for the TPFA scheme, following [20], depends on $\varepsilon$ since the size of the global mesh depends on the size of the rectangles, and the sum of the length of the atypical edges is equal to $\varepsilon$. Under the assumption that $h<\varepsilon$, the most significant term is $O(\sqrt{\varepsilon})$, and it is impossible to get an estimate written in terms of a function of $h$. Quite the contrary, the error estimate obtained in [17] for the hybrid scheme can be expressed as a function of $h$ (this result is generalized in Theorem 8.1), as well as a function of $\varepsilon$. This is a main advantage of working in the dimensionally-heterogeneous domain $D_{\varepsilon, p o l y}$.

9. Numerical experiment. We solve the heat equation (4.1) in a domain $B_{\varepsilon}$ with three vertices and two edges.

In the first series of tests, we take a polynomial function and we choose a suitable right hand side and an appropriate boundary conditions if necessary, so that this function is a solution of the heat equation. Then, the difference between the exact solution and the approximate solution of the partially decomposed problem (7.2) obtained using the finite volume hybrid scheme (7.4) is estimated. The size of the error turns out to correspond to the theoretical predictions, both depending on $h$ and depending on $\varepsilon$.

In the second series of tests, we choose another right hand side and we solve (4.1) without knowing the exact solution. Then the approximate solutions obtained with the size of the mesh $h$ and $h / 2$ are compared. The behavior of the error as a function of $h$ proves to be consistent with the theoretical predictions.

Two examples are examined. The first one is related to a function $f$ that satisfies the requirements of Theorem 6.2. The second one is chosen in a more general context and show that the results of Theorem 6.2 are robust, since the estimate is still valuable if the conditions are not all met.

To begin with, a domain with one node and two edges is considered. Next, a domain with three nodes and two edges.

9.1. One node and two edges. The domain $B_{\varepsilon}, B_{\varepsilon}=\left(\cup_{j=1}^{M} B_{\varepsilon}^{\left(e_{j}\right)}\right) \cup \omega_{\varepsilon}^{1}$ is represented in Figure 9.1-a with $M=2, \theta_{1}=\theta_{2}=1, l_{1}=l_{2}=1$. Let us define

$$
u_{\varepsilon}(x, t)=\left\{\begin{array}{l}
10^{7}(t-0.5)^{5}\left(x_{1}^{\left(e_{1}\right)}-0.5\right)^{3}\left(x_{1}^{\left(e_{1}\right)}-0.9\right)^{3} \\
\text { if } \left.x_{1}^{\left(e_{1}\right)} \in\right] 0.5 ; 0.9[\text { and } t>0.5 \\
10^{7}(t-0.5)^{5}\left(x_{1}^{\left(e_{2}\right)}-0.4\right)^{3}\left(x_{1}^{\left(e_{2}\right)}-0.8\right)^{3} \\
\text { if } \left.x_{1}^{\left(e_{2}\right)} \in\right] 0.4 ; 0.8[\text { and } t>0.5 \\
0 \quad \text { otherwise }
\end{array}\right.
$$

The right hand side $f$ in problem (4.1) is chosen in such a way that $u_{\varepsilon}$ is a solution to this problem corresponding to the value $T=1$. The function $f$ satisfies the requirements of Theorem 6.2.

The difference between $u_{\varepsilon}$ and the approximate solution of (7.2) is computed. To put it more precisely, we compute $\left\|\underline{\hat{x}}_{\varepsilon, \mathcal{T}}^{n}-\underline{u}_{\varepsilon, \delta, d e c, \mathcal{T}}^{n}\right\|_{2, \mathcal{T}}$ with $n$ such that $n k=T=1$, where $\underline{\hat{u}}_{\varepsilon, \mathcal{T}}^{n}$ is the restriction of $\hat{u}_{\varepsilon, \mathcal{T}}^{n}$ to $D_{\varepsilon, \text { poly }}, \hat{u}_{\varepsilon, \mathcal{T}}^{n}$ being a function defined for 
$x \in B_{\varepsilon, \text { poly }}$ by

$\hat{u}_{\varepsilon, \mathcal{T}}^{n}(x)=\left\{\begin{array}{l}u_{\varepsilon}\left(x_{K}, t_{n}\right), x \in K, K \in \mathcal{T}_{1} \\ u_{\varepsilon}\left(x_{i}^{e_{j}}, t_{n}\right), x \in B_{1, j+1}^{d e c, \varepsilon}, x_{1}^{\left(e_{j}\right)} \in\left(x_{i-1 / 2}^{e_{j}}, x_{i+1 / 2}^{e_{j}}\right), i=1, \ldots, N_{j}, j \in\{1,2\} .\end{array}\right.$

We remind that $u_{\varepsilon, \delta, d e c}$ is the solution of $(7.2)$ and $\underline{u}_{\varepsilon, \delta, d e c, \mathcal{T}}^{n}$ is defined in Remark 5 .

Let us evaluate the order of the error of the scheme. We take $\delta \simeq \varepsilon|\ln \varepsilon|$ so that $\left\|u_{\varepsilon}-u_{\varepsilon, \delta, \text { dec }}\right\|_{L^{2}\left(B_{\varepsilon, \text { poly }} \times(0, T)\right)}=O\left(\varepsilon^{J}\right)$, for some $J$, according to Theorem 6.2. So, due to Theorem 8.1, the value of $\left\|\underline{\hat{x}}_{\varepsilon, \mathcal{T}}^{n}-\underline{u}_{\varepsilon, \delta, \operatorname{dec}, \mathcal{T}}^{n}\right\|_{2, \mathcal{T}}$ must be the same as $\left\|e_{\mathcal{T}}^{n}\right\|_{2, \mathcal{T}}$, that is to say $O\left(\frac{h}{\sqrt{\varepsilon}}+k\right)$ according to $(8.25)$.

First, the error is studied as a function of $\varepsilon$. The partially decomposed problem (7.2) is solved for different values of $\varepsilon$ using the hybrid scheme (7.4). For each value of $\varepsilon$, we choose $\delta$ of order $\varepsilon \ln \varepsilon, \varepsilon$ ranging from 0.025 to 0.2 . We get the error estimate between the solution of (7.2) and its numerical approximation with $h=0.01$ and $k=0.002$. All numerical calculations are made with UMFPACK (LU direct method) using the Matlab software. The error is expressed in terms of $\varepsilon$ in logarithmic scale in Figure 9.1-b. The points are perfectly lined up. The slope of the line gives the order of convergence in terms of $\varepsilon$ of the numerical scheme provided that $k$ is very small compared to $\frac{h}{\sqrt{\varepsilon}}$ (it is the case here). If we consider that $h$ is always smaller than $\varepsilon$, then we can expect an error of order $O(\sqrt{\varepsilon})$. And we do obtain a slope equal to 0.5 .
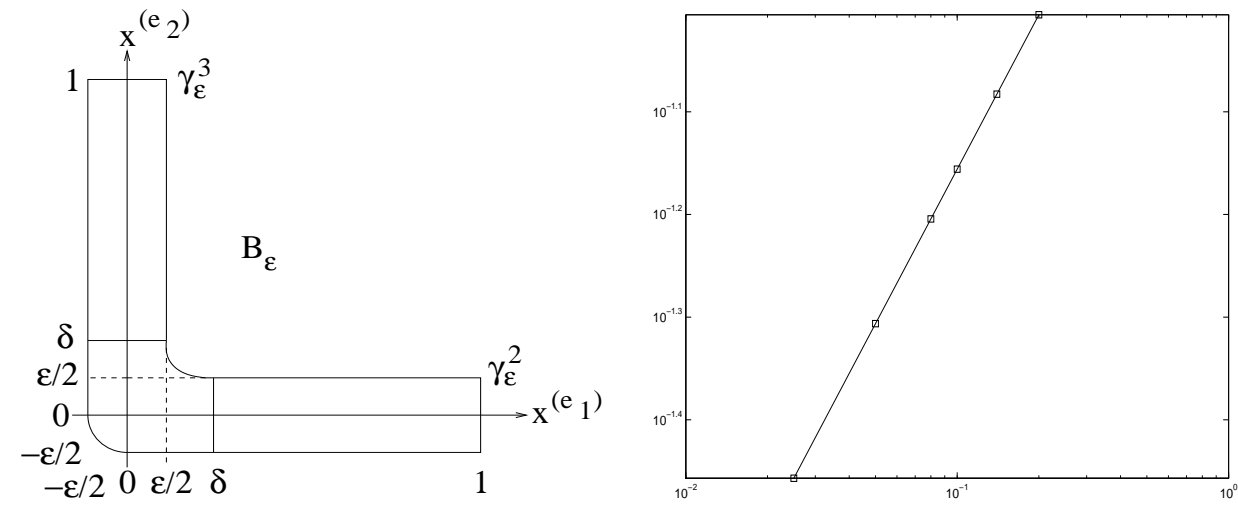

FIG. 9.1. a) The domain $B_{\varepsilon}$ b) Error as a function of $\varepsilon$ when the exact solution is known

Then, we study the error as a function of the space size $h$ while $\varepsilon$ is fixed. To observe the contribution of $h$, we keep $k=0.002$, being small compared to $\frac{h}{\sqrt{\varepsilon}}$. We take $\varepsilon=0.2$ (and $\delta=0.3$ ). In this case the term $\frac{h}{\sqrt{\varepsilon}}$ dominates in the error estimate (with respect to $k$ as well as $\varepsilon^{J}$ ). The error curve (relatively to the variations of $h$ ) is shown in Figure 9.2-a. The slope of the line of least squares (logarithmic scale) is equal to 2.4 and gives an average value of the order of convergence in terms of $h$. The slopes between two successive points are given in Table 9.1. Here $h$ is chosen above the value 0.07 and below 0.175 . For these values the term $\frac{h}{\sqrt{\varepsilon}}$ still dominates in the 
error estimate but it remains a small parameter. On the whole, the results are in good agreement with those predicted. On average, the numerical convergence order is better than the theoretical one. Let us remark that we also observe a difference between the numerical and the theoretically predicted convergence orders for other schemes, for instance the TPFA scheme (see [11] and [18]) solving the Laplace equation. So, the same difference is observed for the heat equation.
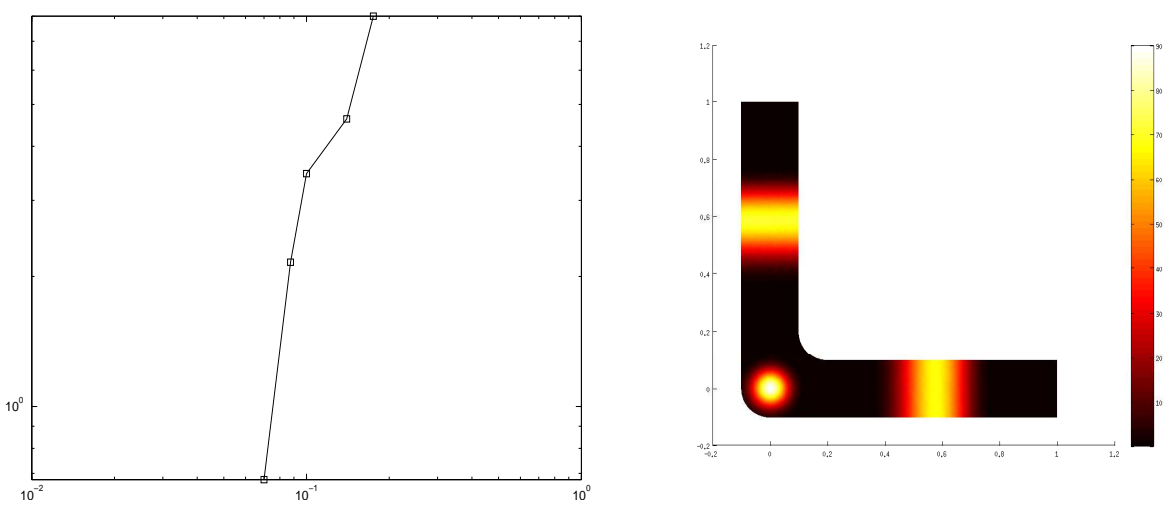

FIG. 9.2. a) Error as a function of $h$ when the exact solution is known b) The right hand side

\begin{tabular}{|c|c|c|c|c|c|}
\hline$h$ & 0.175 & 0.14 & 0.1 & 0.0875 & 0.07 \\
\hline slope & - & 2.45 & 0.87 & 3.53 & 5.19 \\
\hline \multicolumn{7}{|c|}{ TABLE 9.1}
\end{tabular}

Convergence rate when the exact solution is known.

Thereafter, in a second series of tests, we solve (4.1) taking as a right hand the function $f$ which is defined below. The function is best explained by referring to Figure 9.2-b.

$(9.2) f(x, t)=\left\{\begin{array}{l}210^{8}\left(x_{1}^{\left(e_{1}\right)}+2\right)^{2}\left(x_{1}^{\left(e_{2}\right)}+1\right) \exp \left(1 /\left(\left(x_{1}^{\left(e_{1}\right)}\right)^{2}+\left(x_{1}^{\left(e_{2}\right)}\right)^{2}-(0.25)^{2}\right)\right) \\ \text { if } \sqrt{\left(x_{1}^{\left(e_{1}\right)}\right)^{2}+\left(x_{1}^{\left(e_{2}\right)}\right)^{2}}<0.25 \\ 310^{4}\left(5 x_{1}^{\left(e_{1}\right)}\right)^{2} \exp \left(1 /\left(x_{1}^{\left(e_{1}\right)}-0.2\right)\left(x_{1}^{\left(e_{1}\right)}-0.9\right)\right) \\ \text { if } 0.2<x_{1}^{\left(e_{1}\right)}<0.9 \\ 510^{4}\left(3 x_{1}^{\left(e_{2}\right)}\right)^{3} \exp \left(1 /\left(x_{1}^{\left(e_{2}\right)}-0.2\right)(y-0.9)\right) \\ \text { if } 0.2<x_{1}^{\left(e_{2}\right)}<0.9 \\ 0 \quad \text { otherwise }\end{array}\right.$

The function $f$ doesn't satisfy all assumptions of Theorem 6.2 since it isn't constant with respect to $x$ in the neighborhood of the node. It is interesting to see how the scheme behaves in such conditions. We do not know the exact solution of (4.1). The approximate solutions obtained at the time $T=1$ with the sizes of the mesh $h$ and $h / 2$ are compared. As above, we take $\varepsilon=0.2, \delta=0.3$, and $k=0.002$. As mentioned above, we can not observe the convergence rate if $\frac{h}{\sqrt{\varepsilon}}$ is not large enough because there is a threshold effect. We retain only the slope that is equal to 1.56 , which connects the errors obtained for $h=0.175$ (when it is compared with $h=0.0875$ ) 
and $h=0.0875$ (when it is compared with $h=0.04375$ ), and the slope 1.54 which connects the errors obtained by comparing the two solutions calculated for $h=0.35$ (when it is compared with $h=0.175$ ) and $h=0.175$ (As we don't look at the error in terms of $\varepsilon$, it is possible to choose a value of $h$ greater than $\varepsilon$ here). The numerical results are quite consistent with those of the previous test. The order of numerical convergence depending on the size of the mesh is around 2.0.

9.2. Three nodes and two edges. Here, the heat equation is solved in a domain $B_{\varepsilon}$ slightly different from the previous shape. Introduce three nodal domains $\omega_{\varepsilon}^{j}, j \in\{1,2,3\}$, and $B_{\varepsilon}=\left(\cup_{j=1}^{2} B_{\varepsilon}^{\left(e_{j}\right)}\right) \cup\left(\cup_{j=1}^{3} \omega_{\varepsilon}^{j}\right)$. In this case, the asymptotic partial decomposition of domain keeps the two-dimensional in space setting for the heat equation within three pieces $B_{i}^{\varepsilon, \delta}, i \in\{1,2,3\}$, and reduces the dimension to one on the two pieces $e_{1,2}^{d e c, \delta}$ and $e_{1,3}^{d e c, \delta} . B_{\varepsilon}$ is represented in Figure 9.3 with $M=2$, $\theta_{1}=\theta_{2}=1, l_{1}=l_{2}=1$. The length of the segments $e_{12}^{d e c, \delta}$ and $e_{13}^{d e c, \delta}$ is $1-2 \delta$. The ends have a round shape. The exact shape of $B_{i}^{\varepsilon, \delta}$ as a figure constructed as some union (or difference) of ellipses and rectangles is given in Figure 9.3.

We solve
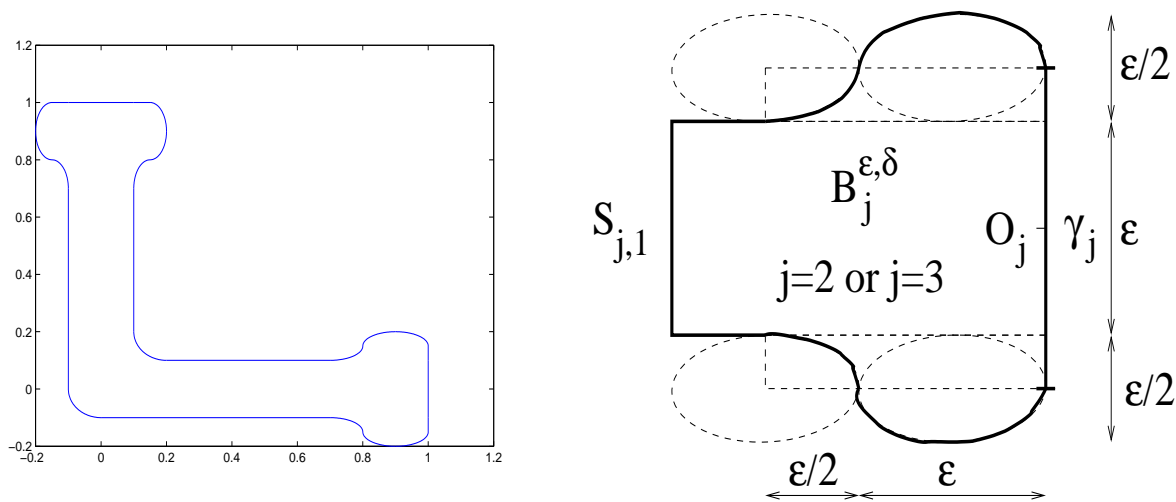

FIG. 9.3. The domain $B_{\varepsilon}$ and the detailed construction of $B_{i}^{\varepsilon, \delta}$.

$$
\left\{\begin{array}{l}
\frac{\partial u_{\varepsilon}}{\partial t}(x, t)-\triangle u_{\varepsilon}(x, t)=f(x, t), x \in B_{\varepsilon}, t \in(0, T) \\
u_{\varepsilon}(x, t)=0, x \in \gamma_{j}, j \in\{2,3\}, t \in(0, T) \\
\frac{\partial u_{\varepsilon}(x, t)}{\partial n}=g(x, t), x \in \partial B_{\varepsilon} \backslash\left(\cup_{j=2}^{3} \gamma_{j}\right), t \in(0, T)
\end{array}\right.
$$

The functions $f$ and $g$ are defined so that the following function $u_{\varepsilon}$ is a solution to $(9.3)$.

$$
u_{\varepsilon}(x, t)= \begin{cases}100 t\left(-4\left(x_{1}^{e_{1}}\right)^{3}+6\left(x_{1}^{e_{1}}\right)^{2}-3 x_{1}^{e_{1}}+1\right) & \text { if } x \geq 0.5 \\ 100 t\left(-4\left(x_{1}^{e_{2}}\right)^{3}+6\left(x_{1}^{e_{2}}\right)^{2}-3 x_{1}^{e_{2}}+1\right) & \text { if } y \geq 0.5 \\ 50 t & \text { else }\end{cases}
$$

As far as the Neumann boundary condition is concerned, the function $g$ is zero in (4.1) and it is not in (9.3). The Dirichlet condition part of the boundary is defined by $\gamma_{2}=\left\{x_{1}^{\left(e_{1}\right)}=1, x_{2}^{\left(e_{1}\right)} \in\left(-\frac{3 \varepsilon}{4}, \frac{3 \varepsilon}{4}\right)\right\}$, and $\gamma_{3}=\left\{x_{1}^{\left(e_{2}\right)}=1, x_{2}^{\left(e_{2}\right)} \in\left(-\frac{3 \varepsilon}{4}, \frac{3 \varepsilon}{4}\right)\right\}$. The previous theoretical result still remains valid with any kind of boundary conditions 
and the right hand side, provided that the solution of the heat equation is regular. As the solution (9.4) of (9.3) is regular, we expect to have the same error estimates as those of Theorem 6.2 and Theorem 8.1.

The hybrid finite volume scheme is defined on a grid constructed with three admissible meshes $\mathcal{T}_{i}, i \in\{1,2,3\}$ for the regions $B_{i, \text { poly }}^{\varepsilon, \delta}, i \in\{1,2,3\}$, and with two regular one-dimensional meshes with the same size for the segments $e_{1, j}^{\text {dec, },}, j \in\{2,3\}$. The approximate solution is computed at the time $T=1$ and compared with (9.4).

First, we choose $h=0.01$ and $k=0.01$. Then we trace the error curve as a function of $\varepsilon, \varepsilon$ ranging from 0.025 to 0.2 . For each value of $\varepsilon, \delta$ is chosen close to $\varepsilon|\ln \varepsilon|$. We compute the error for a cloud of points (26 points) and apply the least squares with the logarithmic scale to plot the line. Recall that we calculate the solution at time $T=1$, which corresponds to $n=100$ iterations, since $k=0.01$ (see Figure 9.4 -a). The scatter after $n=500$ iterations with $k=0.002$ is also represented (see Figure 9.4-b). The slope obtained for $n=100$ is 0.48 . It is 0.52 for $n=500$. So the result is consistent with theoretical predictions.
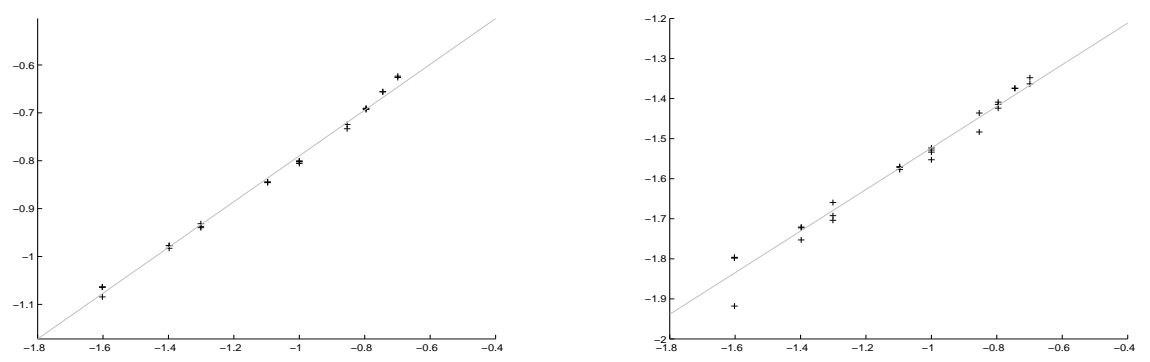

FIG. 9.4. Error between the exact solution and its approximation as a function of $\varepsilon$ a) $n=100$ b) $n=500$

In turn, we assume that $\varepsilon$ is fixed to observe the dependence of the error on $h$. We choose $k=0.002$, small compared to $\frac{h}{\sqrt{\varepsilon}}$. We take $\varepsilon=0.2$ (and $\delta=0.3$ ). The curve is shown in Figure 9.5-a. The slope of the line of least squares (logarithmic scale) gives an average value of the order of convergence in terms of $h$, we find 1.6. To put it more precisely, the slope between two successive points is given Table 9.2 and the numerical order of convergence proves to be between 0.92 and 2.02 .

\begin{tabular}{|c|c|c|c|c|}
\hline$h$ & 0.2 & 0.1 & 0.08 & 0.05 \\
\hline slope & - & 1.60 & 0.92 & 2.02 \\
\hline \multicolumn{6}{|c}{ TABLE 9.2}
\end{tabular}

Convergence rate when the exact solution is known.

After all, (4.1) is solved by keeping the Dirichlet condition on $\gamma_{j}$, as in (9.3). The 

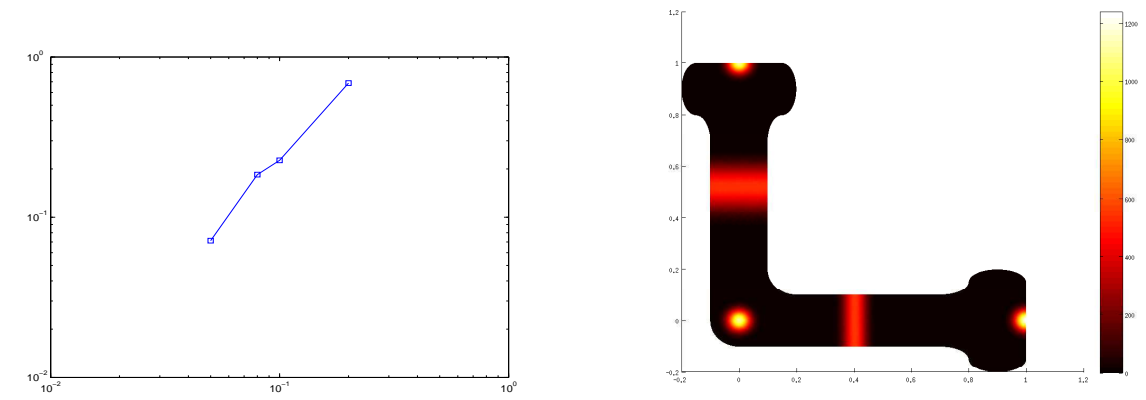

FIG. 9.5. a) Error as a function of $h$ when the exact solution is known b) Second member when the exact solution is not known

function $f$ is defined below and represented in Figure 9.5-b.

$f(x, y)= \begin{cases}0.210^{14}(x+2)^{2}(y+1) \exp \left(1 /\left(x^{2}+y^{2}-(0.2)^{2}\right)\right) & \text { if } \sqrt{x^{2}+y^{2}}<0.2 \\ 10^{13}(x+2)^{2}(y+1) \exp \left(1 /\left((x-1)^{2}+y^{2}-(0.2)^{2}\right)\right) & \text { if } \sqrt{(x-1)^{2}+y^{2}}<0.2 \\ 10^{13}(x+2)^{2}(y+1) \exp \left(1 /\left(x^{2}+(y-1)^{2}-(0.2)^{2}\right)\right) & \text { if } \sqrt{x^{2}+(y-1)^{2}}<0.2 \\ 10^{13}(5 x)^{2} \exp (1 /(x-0.2)(x-0.6)) & \text { if } 0.2<x<0.6 \\ 10^{7}(3 y)^{3} \exp (1 /(y-0.2)(y-0.8)) & \text { if } 0.2<y<0.8 \\ 0 & \text { else }\end{cases}$

The exact solution is unknown. The approximate solutions obtained at the time $T=1$ with the sizes of the mesh $h$ and $h / 2$ are compared. As above, we take $\varepsilon=0.2$, $\delta=0.3$, and $k=0.002$. The order of numerical convergence depending on the mesh size turns to be equal to 1.96. This result is quite consistent with those of the theoretical predictions.

10. Conclusion. A finite volumes numerical scheme of hybrid dimension for solving the heat equation set in thin rod structures is considered and its overall error is estimated. This equation may be used for the modeling of the diffusion process in a net of channels or cylindrical vessels. Such problems arrise in some biomathematical applications [19]. The hybrid dimension scheme allows to reduce considerably the computational cost of the problem keeping high precision.

Acknowledgments. The present work is partially supported by the Research Federative Structures MODMAD FED 4169 and FR CNRS 3490, by the FrenchGerman grant PROCOPE EGIDE 28481WB "Homogenization based optimization for elasticity on the network of beams", by the grant number 14-11-00306 of Russian Scientific Fondation and by LABEX MILYON (ANR-10-LABX-0070) of University of Lyon, within the program "Investissements d'Avenir" (ANR-11-IDEX-0007) operated by the French National Research Agency (ANR).

\section{REFERENCES}

[1] S. Agmon, A. Douglis, L. Nirenberg. Estimates near the boundary for solutions of elliptic partial differential equations satisfying general boundary conditions. I,II Comm. Pure Appl. Math., 12(4):623-723, 1959, 17(1): 35-92, 1964. 
[2] N.S.Bakhvalov, G.P.Panasenko, Homogenisation: Averaging Processes in Periodic Media, Nauka, Moscow, 1984 (in Russian), Engl.Transl. Kluwer, Dordrecht/Boston/London, 1989, $366 \mathrm{pp}$.

[3] S.Marusic, E.Marusic-Paloka, Reduction of dimension for parabolic equations via two-scale convergence, Proceedings of ApplMat 99, Dubrovnik, 1999, Department of Mathematics, University of Zagreb, 2001, 155-164.

[4] O.A.Oleinik,G.A.Yosifian. On the asymptotic behaviour at infinity of solutions in linear elasticity. Arch. Rational Mech. Anal. 78, 1982,2953. Downloaded by

[5] G.P. Panasenko, Method of asymptotic partial decomposition of domain, Mathematical Models and Methods in Applied Sciences , 8, 1, 1998, 139-156.

[6] G.P. Panasenko, Multi-Scale Modelling for Structures and Composites, Springer, Dordrecht, 2005, 398 pp.

[7] G.Panasenko, Method of asymptotic partial domain decomposition for non-steady problems: heat equation on a thin structure, Journal Math. Comm., 2014, in print.

[8] G.Panasenko, R.Stavre, Asymptotic analysis of a viscous fluid-thin plate interaction: periodic flow, Mathematical Models and Methods in Applied Sciences, 24,7, 2014, in print.

[9] G.Panasenko, M.-C.Viallon, The finite volume implementation of the partial asymptotic domain decomposition, Applicable Analysis, 87,12, 2008, 1397-1424.

[10] G.Panasenko, M.-C.Viallon, Error estimate in the finite volume approximation of the partial asymptotic domain decomposition, Math. Methods in the Applied Sciences, 36,14, 2013, 1892-1917; Doi:10.1002/mma.2735

[11] K. Domelevo, and P. Omnes. A finite volume method for the Laplace equation on almost arbitrary two-dimensional grids. M2AN, 39 (6) : 1203-1249, 2005.

[12] F. Fontvieille, G. Panasenko and J. Pousin. FEM implementation for the asymptotic partial decomposition. Applicable Analysis, 86 (5) : 519-536, $200 \%$.

[13] G.P. Panasenko. Asymptotic solutions of the elasticity theory system of equations for lattice and skeletal structures. Math.Sb.,183(1):89-113, 1992 (in Russian). English transl. by AMS in Russian Acad. Sci. Sbornik Math. 75(1):85-110, 1993.

[14] G.P. Panasenko. Method of asymptotic partial decomposition of rod structures. International Journal of Computational, Civil and Structural Engineering (Begel House Publ.) 1(2):5770, 2000.

[15] G.P. Panasenko. Asymptotic expansion of the solution of Navier-Stokes equation in tube structure and partial asymptotic decomposition of the domain, Applicable Analysis an International Journal, 76(3-4): 363-381, 2000.

[16] V.A.Solonnikov. On boundary value problems for linear parabolic systems of general form, Trudy Mat. Inst. Steklov 83: 1-162, 1965, English transl. Proc. Math. Inst. Steklov, 83, 1965.

[17] M.-C. Viallon. Error estimate for a 1D-2D finite volume scheme. Comparison with a standard scheme on a 2D non-admissible mesh. C. R. Acad. Sci. Paris, Ser.I, 351 (2013) 47-51.

[18] R. Eymard, T. Gallouet, R. Herbin, Finite Volume methods, Handbook of Numerical Analysis. Vol VII, 2000, p. 713-1020, Editors : P.G. Ciarlet and J.L. Lions.

[19] L. Formaggia, A. Quarteroni and A. Veniziani. Cardiovascular Mathematics, Series: Modeling Simulation and Applications, vol. 1, Springer, 2009

[20] R. Cautrés, R. Herbin and F. Hubert. The Lions domain decomposition algorithm on non matching cell-centred finite volume meshes. IMA Journal of Numerical Analysis, Vol. 24, pp. 465-490, (2004) 